\title{
Rolling Stability of a Power-Generating Tumbleweed Rover
}

\author{
François Robert Hogan* \\ McGill University, Montreal, Quebec H3A OC3, Canada \\ James Richard Forbes \pm \\ University of Michigan, Ann Arbor, Michigan 48109-2140 \\ and \\ Timothy David Barfoot \\ University of Toronto, Toronto, Ontario M3H 5T6, Canada
}

\begin{abstract}
In this paper, the rolling stability of a power-generating tumbleweed rover is investigated. The nonlinear equations of motion of the rover capture the nonholonomic nature of rolling without slipping, the external wind force, and energy dissipation due to rolling friction and power generation. To assess stability of the system as it rolls about a preferred axis of rotation, Lyapunov's indirect method is employed. When the shell of the tumbleweed rover has a uniform mass distribution, Lyapunov's indirect method predicts instability; when the inertia about a preferred axis of rotation is increased, Lyapunov's indirect method predicts stability. Numerical simulation results of both the linearized and nonlinear equations of motion confirm both the instability and stability predictions of Lyapunov's indirect method.
\end{abstract}

\begin{tabular}{|c|c|c|}
\hline \multicolumn{3}{|r|}{ Nomenclature } \\
\hline$A$ & $=$ & frontal area of rover, $\mathrm{m}^{2}$ \\
\hline$b_{p}$ & $=$ & $\begin{array}{l}\text { damping coefficient associated with power generation, } \\
\left(\mathrm{kg} \cdot \mathrm{m}^{2}\right) / \mathrm{s}\end{array}$ \\
\hline$b_{r}$ & $=$ & $\begin{array}{l}\text { damping coefficient associated with translational rolling } \\
\text { friction, }\left(\mathrm{kg} \cdot \mathrm{m}^{2}\right) / \mathrm{s}\end{array}$ \\
\hline$b_{s}$ & $=$ & $\begin{array}{l}\text { damping coefficient associated with spinning rolling } \\
\text { friction, }\left(\mathrm{kg} \cdot \mathrm{m}^{2}\right) / \mathrm{s}\end{array}$ \\
\hline $\mathbf{C}_{b a}$ & $=$ & $\begin{array}{l}\text { direction cosine matrix transforming the components of } \\
v \text { from } \mathcal{F}_{a} \text { to } \mathcal{F}_{b} \text {, that is, } \mathbf{v}_{b} \text { equal to } \mathbf{C}_{b a} \mathbf{v}_{a}\end{array}$ \\
\hline$C_{d}$ & $=$ & drag coefficient of sphere \\
\hline $\mathbf{c}_{p}^{\mathcal{P} p}$ & $=$ & $\begin{array}{l}\text { first moment of mass of pendulum relative to } \mathcal{F}_{p} \\
\text { expressed in } \mathcal{F}_{p}, \mathrm{~kg} \cdot \mathrm{m}\end{array}$ \\
\hline $\mathcal{F}_{a}$ & $=$ & $\begin{array}{l}\text { generic reference frame "a" associated with basis } \\
\text { vectors }\left\{\boldsymbol{a}^{1}, \boldsymbol{a}^{2}, \boldsymbol{a}^{3}\right\}\end{array}$ \\
\hline$f^{w}$ & $=$ & aerodynamic force applied on system due to wind, $\mathrm{N}$ \\
\hline$g$ & $=$ & gravitational acceleration constant, $\mathrm{m} / \mathrm{s}^{2}$ \\
\hline $\mathbf{J}_{p}^{\mathcal{P} p}$ & $=$ & $\begin{array}{l}\text { second moment of mass of pendulum relative to } \mathcal{F}_{p} \\
\text { expressed in } \mathcal{F}_{p}, \mathrm{~kg} \cdot \mathrm{m}^{2}\end{array}$ \\
\hline $\mathbf{J}_{s}^{\mathcal{S} s}$ & $=$ & $\begin{array}{l}\text { second moment of mass of sphere relative to } \mathcal{F}_{s} \\
\text { expressed in } \mathcal{F}_{s}, \mathrm{~kg} \cdot \mathrm{m}^{2}\end{array}$ \\
\hline$\ell_{\mathcal{P R}}$ & $=$ & length of pendulum rod, $\mathrm{kg}$ \\
\hline$m_{\mathcal{P}}$ & $=$ & mass of pendulum, $\mathrm{kg}$ \\
\hline$m_{\mathcal{P A}}$ & $=$ & mass of payload, $\mathrm{kg}$ \\
\hline$m_{\mathcal{P R}}$ & $=$ & mass of pendulum rod, $\mathrm{kg}$ \\
\hline$m_{\mathcal{S}}$ & $=$ & mass of sphere, $\mathrm{kg}$ \\
\hline $\mathbf{q}$ & $=$ & $\begin{array}{l}{\left[\begin{array}{ll}\mathbf{r}_{i}^{s i \top} & \boldsymbol{\theta}_{i}^{s i \top}\end{array} \boldsymbol{\theta}_{i}^{p i \top}\right]^{\top} \text { equal to dependent generalized }} \\
\text { coordinates }\end{array}$ \\
\hline$\hat{\mathbf{q}}$ & $=$ & $\begin{array}{ll}{\left[\begin{array}{ll}\boldsymbol{\theta}^{s i \top} & \phi\end{array}\right]^{\top} \text { equal to reduced generalized coordinates, }} \\
\mathrm{rad} / \mathrm{s}\end{array}$ \\
\hline
\end{tabular}

Received 7 November 2013; revision received 14 July 2014; accepted for publication 19 July 2014; published online 20 October 2014. Copyright (C) 2014 by François Robert Hogan, James Richard Forbes, and Timothy David Barfoot. Published by the American Institute of Aeronautics and Astronautics, Inc., with permission. Copies of this paper may be made for personal or internal use, on condition that the copier pay the $\$ 10.00$ per-copy fee to the Copyright Clearance Center, Inc., 222 Rosewood Drive, Danvers, MA 01923; include the code 1533-6794/14 and \$10.00 in correspondence with the CCC.

*Master's Candidate, Department of Mechanical Engineering; francois .hogan@mail.mcgill.ca.

${ }^{\dagger}$ Assistant Professor, Department of Aerospace Engineering, FXB Building, 1320 Beal Ave.; forbesrj@umich.edu.

₹Associate Professor, Institute for Aerospace Studies; tim.barfoot@ utoronto.ca.

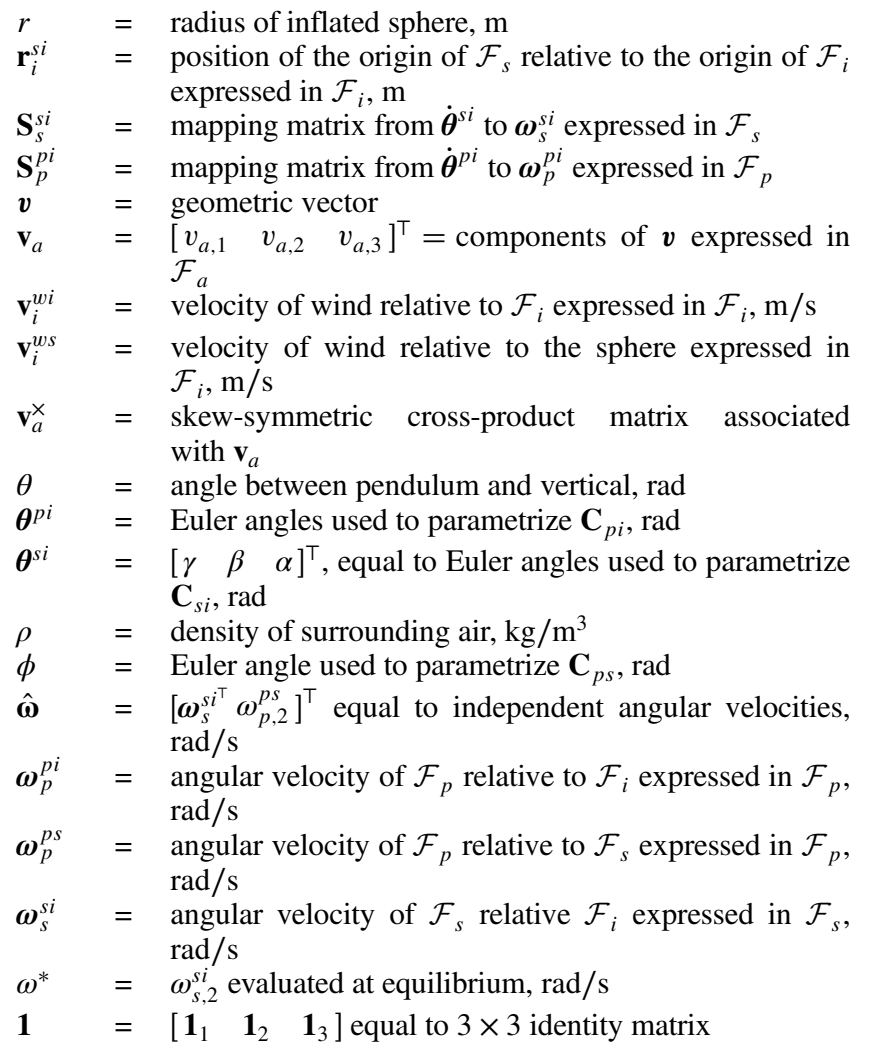

\section{Introduction}

$\mathbf{H}$ ISTORICALLY, the exploration of the Martian surface has been performed by stationary landers or wheeled vehicles. The current exploration strategy adopted by space agencies is to target a landing zone of interest, deploy a rover to conduct experiments, and collect data in the vicinity of the targeted area. These wheeled rovers are equipped with an impressive array of scientific instruments that may be used to monitor the Martian climate and characterize its geology. Wheeled vehicles are ideal for performing scientific experiments, but present a major drawback to planetary exploration: a slow exploration velocity. The speed of wheeled rovers is limited by communication and computational delays, rocker-bogie suspension designs, and available power [1]. Because many areas of scientific 
interest may lie several kilometers away from landing sites, the limited mobility of wheeled vehicles presents an important problem.

In an effort to address this problem, radically different rover designs have been investigated to achieve a higher exploration velocity of Mars. A design of interest that shows great promise is the tumbleweed rover [2]. A wind-powered tumbleweed rover is a large sphere-shaped structure that uses Martian winds as its means of propulsion. Consider a mission scenario where a wheeled rover equipped with one (or perhaps more) tumbleweed rovers onboard is rather far from a site of interest. Before sending the wheeled rover to the site, a tumbleweed rover could be deployed under favorable wind conditions to perform a scouting mission [2]. The purpose of the scouting mission would be to collect preliminary data about the site of interest as well as information about the route from the wheeled rover's current position to the site [3] . This information would be used to assess the risk of the wheeled rover going to the site, as well as the scientific interest of the site itself. In recent years, the development of new tumbleweed rover concepts has been an active area of research. A promising concept, detailed in [4], integrates an instrument payload inside a large inflatable ball. This large inflatable ball is blown by surface winds much like a terrestrial tumbleweed. This tumbleweed rover concept was tested in Greenland and Antarctica, where it successfully travelled over $100 \mathrm{~km}$ while transmitting temperature and pressure information back to a base every $30 \mathrm{~min}$. This tumbleweed design made no attempt to control the speed and trajectory of the rover, and the onboard instruments were powered using a battery pack.

Other researchers have favored a deployable open structure over an inflated ball design and have developed many different concepts, including box-kite, dandelion, eggbeater dandelion, and tumble-cup configurations $[5,6]$. Open-structure designs were shown to have superior aerodynamic properties over inflated sphere designs and allow direct contact with the Martian environment for scientific experimentation. However, a drawback of these designs is that they offer a reduced available payload area for scientific equipment $[\underline{7}, \underline{8}]$. Open-structure tumbleweed rover designs that integrate interior sails to capture wind forces are extensively studied in [9-11].

A power-generating tumbleweed rover, which is the focus of this paper, was developed in $[\underline{8}, 12]$. A power-generating tumbleweed rover is an inflated ball that integrates an internal pendulumgenerator system that converts the kinetic energy of the pendulum into electric energy. The electric energy produced by the generator is stored inside a battery and can either be used by the tumbleweed rover to power the scientific equipment onboard or, if the generator is operated as a motor, to drive the vehicle forward should wind not be available. The autonomy provided by the pendulum-generator system along with the large available area for scientific payloads make this design an ideal candidate for Mars exploration scouting missions. For the pendulum-generator system to produce electrical energy in an efficient way, it is crucial that the rover rolls about a preferred axis of rotation. However, the roll stability predicted by previous numerical simulations [12] has not been observed in experimental trials [8]. Field tests performed on the power-generating tumbleweed rover of [12] have demonstrated that it rolls in an unstable and chaotic manner, as depicted in Fig. 1. The discrepancy between numerical simulation results and experimental results is believed to be a result of neglecting tumbleweed rover components in the equations of motion. These components effectively increase the moment of inertia of the tumbleweed rover about the nonpreferred

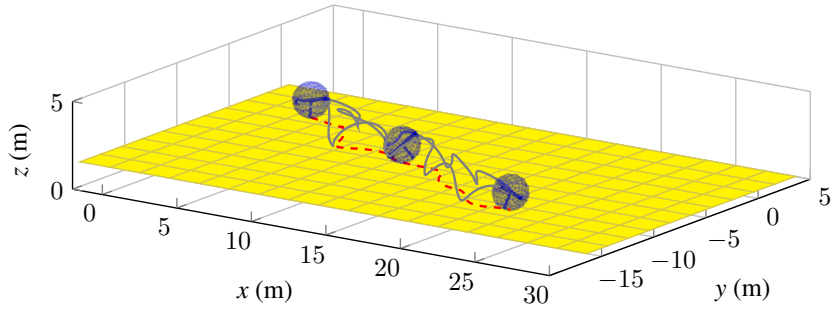

Fig. 1 Unstable rolling of tumbleweed rover. axis of rotation. It is this discrepancy that sparked interest in better understanding the influence of the moment of inertia of the structure on the stability of the power-generating tumbleweed rover.

The majority of research conducted on tumbleweed rovers makes use of simplified two-dimensional quasi-static models [13] and threedimensional models where the rover is assumed to be a spherical shell [14]. With the tumbleweed rover design investigated in this paper, these assumptions cannot be made because the pendulum system has a large impact on the motion and stability of the system. Analytical models for a sphere integrating a pendulum system have been studied in $[12,15-19]$. The three-dimensional rover model used in this paper is developed in [12], where the equations of motion of the rover are derived from first principles using a Lagrangian approach.

This paper's aim is to perform a rigorous stability analysis of the power-generating tumbleweed rover. Although extensive work has been performed on the modeling and control of spherical robots [2025], the rolling stability of these systems has not been explicitly studied. Rolling stability is defined as asymptotically stable rolling about the preferred axis of rotation such that the perturbations associated with the rover's roll, yaw, roll rate, and yaw rate decay to zero with time. In [12], it is shown that the power-generating tumbleweed rover is stable in the sense of Lyapunov when released with nonzero velocity and rolling to a stop (i.e., zero velocity) when wind is not present, but the rolling stability of the rover in the presence of wind has yet to be considered. In this paper, Lyapunov's indirect method is used to investigate the stability of the tumbleweed rover when rolling about the preferred axis of rotation. This paper also analyzes tumbleweed rover configurations where the inertia of the rover about the preferred axis of rotation is increased, which has been proposed in $[\underline{4}, \underline{8}]$ as a solution to the aforementioned stability problems. Finally, the proposed stability analysis method is validated through a numerical simulation study, where it is shown that a rover with a spherical shell with a uniform mass distribution does not roll stably, and that the addition of mass about the preferred axis of rotation leads to stable rolling, as depicted in Fig. 2 .

\section{Preliminaries}

\section{A. Notation}

A geometric vector $\boldsymbol{v}$, which is a frame invariant entity, can be expressed in the reference frame $\mathcal{F}_{a}$ :

$$
\boldsymbol{v}=\boldsymbol{a}^{1} v_{a, 1}+\boldsymbol{a}^{2} v_{a, 2}+\boldsymbol{a}^{3} v_{a, 3}
$$

where $v_{a, 1}, v_{a, 2}$, and $v_{a, 3}$ are scalar components of $\boldsymbol{v}$, and $\boldsymbol{a}^{1}, \boldsymbol{a}^{2}$, and $\boldsymbol{a}^{3}$ are the unit vectors associated with $\mathcal{F}_{a}$. Throughout this paper, a reference frame is defined as a triad of orthogonal basis vectors ([26] pp. 6-7) and lowercase boldface roman italic symbols are reserved for geometric vectors. The scalar components of $\boldsymbol{v}$ expressed in $\mathcal{F}_{a}$ can be represented as a column matrix:

$$
\mathbf{v}_{a}=\left[\begin{array}{l}
v_{a, 1} \\
v_{a, 2} \\
v_{a, 3}
\end{array}\right]
$$

Notice that a right subscript is used to denote what frame the geometric vector is expressed in. It is possible to transform the components of a geometric vector from $\mathcal{F}_{a}$ to $\mathcal{F}_{b}$ by using the direction cosine matrix $\mathbf{C}_{b a}$ :

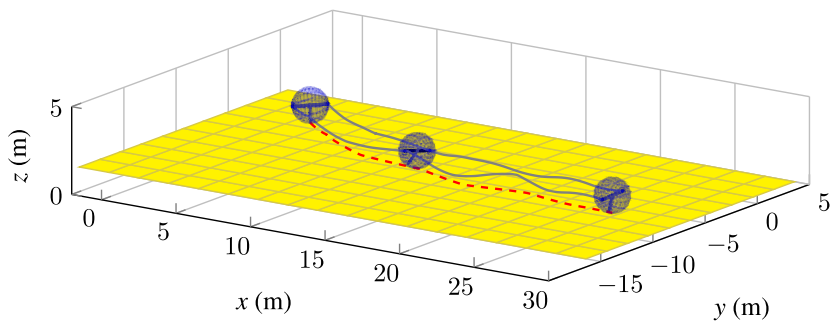

Fig. 2 Stable rolling of tumbleweed rover. 


$$
\mathbf{v}_{b}=\mathbf{C}_{b a} \mathbf{v}_{a}
$$

The column matrix $\mathbf{v}_{b}$ contains the scalar components of $\boldsymbol{v}$ when expressed in reference frame $\mathcal{F}_{b}$, where $\boldsymbol{b}^{1}, \boldsymbol{b}^{2}$, and $\boldsymbol{b}^{3}$ are the unit vectors associated with $\mathcal{F}_{b}$. The cross product between $\boldsymbol{u}$ and $\boldsymbol{v}$ can be expressed in reference frame $\mathcal{F}_{a}$ as

$$
\begin{aligned}
\boldsymbol{w}= & \boldsymbol{u} \times \boldsymbol{v} \\
= & \underbrace{\left(u_{a, 2} v_{a, 3}-u_{a, 3} v_{a, 2}\right)}_{w_{a, 1}} \boldsymbol{a}^{1}+\underbrace{\left(u_{a, 3} v_{a, 1}-u_{a, 1} v_{a, 3}\right)}_{w_{a, 2}} \boldsymbol{a}^{2} \\
& +\underbrace{\left(u_{a, 1} v_{a, 2}-u_{a, 2} v_{a, 1}\right)}_{w_{a, 3}} \boldsymbol{a}^{3}
\end{aligned}
$$

In component form, the cross product can be represented using a $3 \times 3$ skew-symmetric matrix:

$$
\mathbf{w}_{a}=\mathbf{u}_{a}^{\times} \mathbf{v}_{a}
$$

where $\mathbf{u}_{a}=\left[\begin{array}{lll}u_{a, 1} & u_{a, 2} & u_{a, 3}\end{array}\right]^{\top}$ and ([27] p. 85)

$$
\mathbf{u}_{a}^{\times}=\left[\begin{array}{ccc}
0 & -u_{a, 3} & u_{a, 2} \\
u_{a, 3} & 0 & -u_{a, 1} \\
-u_{a, 2} & u_{a, 1} & 0
\end{array}\right]
$$

In this paper, the terms $\mathbf{1}_{1}=\left[\begin{array}{lll}1 & 0 & 0\end{array}\right]^{\top}, \mathbf{1}_{2}=\left[\begin{array}{lll}0 & 1 & 0\end{array}\right]^{\top}$, and $\mathbf{1}_{3}=\left[\begin{array}{lll}0 & 0 & 1\end{array}\right]^{\top}$ are used to express the columns of the identity matrix 1.

\section{B. Model Setup}

Consider the tumbleweed rover model displayed in Fig. 3a. A central rod is fixed to the sides of the inflated sphere. Attached to the middle of the central rod is a pendulum rod and a payload. In this paper, the pendulum rod and the payload components will be referred to as the pendulum system. At the central rod-connecting rod joint is a power generator designed to harvest electric energy as the tumbleweed rover rotates, and the pendulum induces a torque on the generator. The external mass distribution about the preferred axis of rotation, referred to as the spherical annulus, can be seen in Fig. $3 \mathrm{~b}$. Note that the central rod component and the spherical annulus were both neglected in the model derived in [12]. The outside of the sphere is assumed to have small protrusions, similar to the design described in [4], that will prevent the rover from sliding and that will contribute to the inertia of the spherical annulus.

\section{Reference Frames}

To describe the motion of the sphere and pendulum, it is useful to define three reference frames: an inertial frame fixed to the ground,

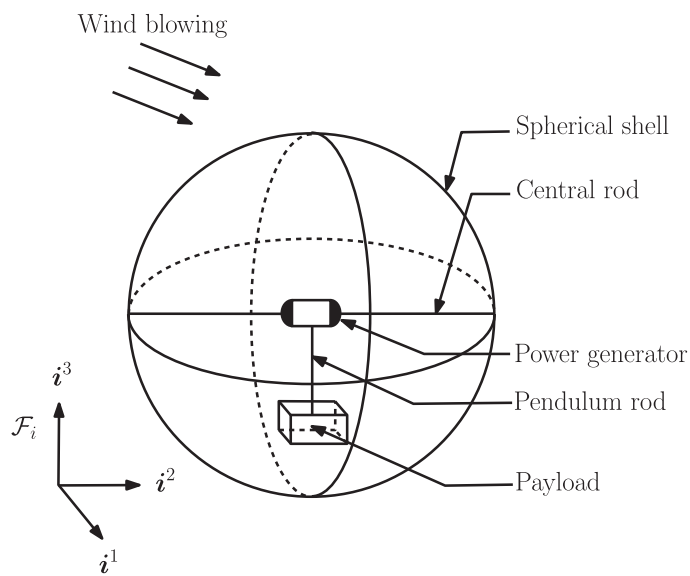

a) Internal view denoted $\mathcal{F}_{i}$; a body frame fixed to the center of the rolling sphere, denoted $\mathcal{F}_{s}$; and a body frame fixed to the pendulum, denoted $\mathcal{F}_{p}$. In Fig. $4 \mathrm{a}$, it can be seen that the $\boldsymbol{s}^{1}-\boldsymbol{s}^{3}$ plane and the $\boldsymbol{p}^{1}-\boldsymbol{p}^{3}$ plane are coincident because the pendulum is constrained to rotate about the $\left(s^{2}, p^{2}\right)$ axis.

The Euler angles $\boldsymbol{\theta}^{s i}$ are used to parameterize the direction cosine matrix $\mathbf{C}_{s i}$ that transforms the components of a geometric vector resolved in $\mathcal{F}_{i}$ to the components of that same geometric vector resolved in $\mathcal{F}_{s}$, and the Euler angles $\boldsymbol{\theta}^{p i}$ are used to parameterize the direction cosine matrix $\mathbf{C}_{p i}$ that transforms the components of a geometric vector resolved in $\mathcal{F}_{i}$ to the components of that same geometric vector resolved in $\mathcal{F}_{p}$.

\section{Equations of Motion}

An overview of the derivation of the equations of motion is presented in the Appendix (see [12] for a detailed derivation). The equations of motion of the tumbleweed rover model depicted in Fig. $\underline{3}$ are

$$
\hat{\mathbf{M}} \dot{\hat{\boldsymbol{\omega}}}+\hat{\boldsymbol{\tau}}^{\mathrm{non}}=\hat{\boldsymbol{\tau}}^{\mathrm{ext}}
$$

with

$$
\hat{\boldsymbol{\omega}}=\left[\begin{array}{ll}
\boldsymbol{\omega}_{s}^{s i^{\top}} & \omega_{p, 2}^{p s}
\end{array}\right]^{\top}
$$

where $\boldsymbol{\omega}_{s}^{s i}$ describes the angular velocity of $\mathcal{F}_{s}$ relative to $\mathcal{F}_{i}$ expressed in $\mathcal{F}_{s}$, and $\omega_{p, 2}^{p s}$ is the second component of $\omega_{p}^{p s}$, which describes the angular velocity of $\mathcal{F}_{p}$ relative to $\mathcal{F}_{s}$ expressed in $\mathcal{F}_{p}$. Additionally,

$$
\begin{aligned}
& \hat{\mathbf{M}}=\Delta \mathbf{M \Pi}, \\
& \hat{\boldsymbol{\tau}}^{\mathrm{non}}=\boldsymbol{\Delta}\left(\mathbf{M} \dot{\boldsymbol{\Pi}} \hat{\boldsymbol{\omega}}+\boldsymbol{\nu}^{\times} \mathbf{M} \nu+\mathbf{a}\right), \quad \text { and } \quad \hat{\boldsymbol{\tau}}^{\mathrm{ext}}=\Delta \boldsymbol{\tau}^{\mathrm{ext}}
\end{aligned}
$$

where the matrices $\Delta, \mathbf{M}, \boldsymbol{\Pi}, \dot{\mathbf{\Pi}}, \nu, \nu^{\times}$and $\mathbf{a}$ are given by Eqs. (A5-A11) in the Appendix.

\section{Stability Analysis}

Because the pendulum is constrained to rotate about the $s^{2}$ axis, the power generator will produce electrical energy in an efficient way when the rover is rotating about the $s^{2}$ axis, referred to as the preferred axis. Because this configuration is desired, it is important to investigate the stability of the rover rolling about the preferred axis, also called the preferred configuration or equilibrium state. In this section, the perturbed dynamics of the rover about the preferred configuration will be derived and the stability of the equilibrium state will be studied using Lyapunov's indirect method. The stability analysis is the main contribution of this paper and is motivated by the discrepancy between the results obtained in [8] and [12]. The analysis carried

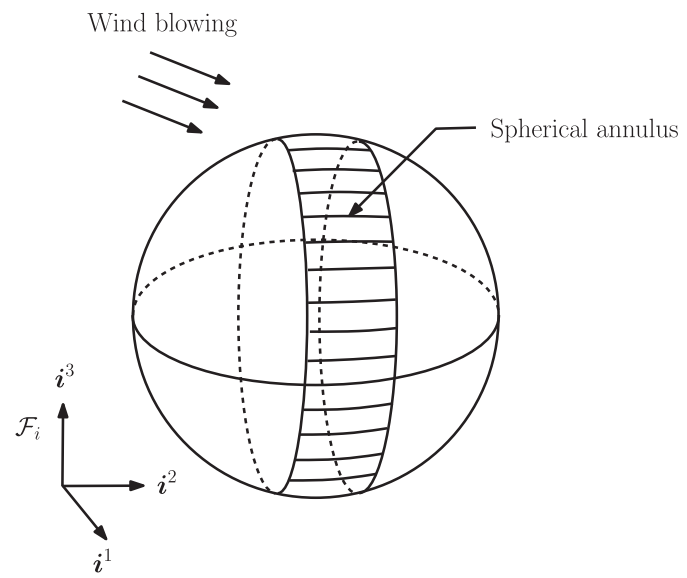

b) External view

Fig. 3 Tumbleweed rover model. 


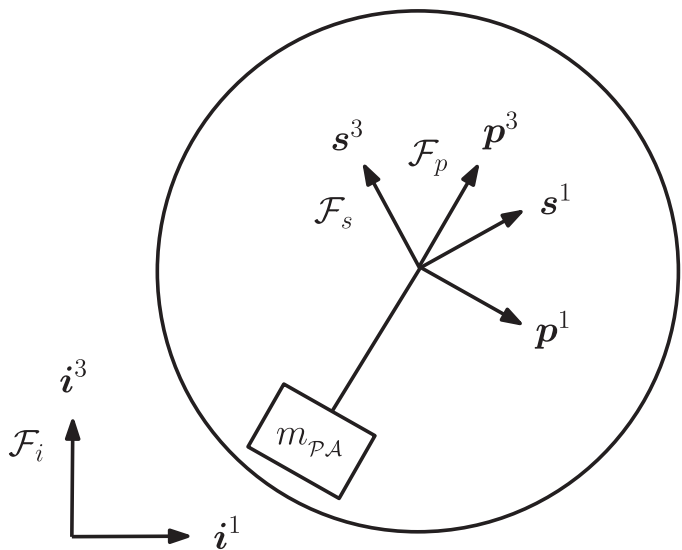

a) Side view

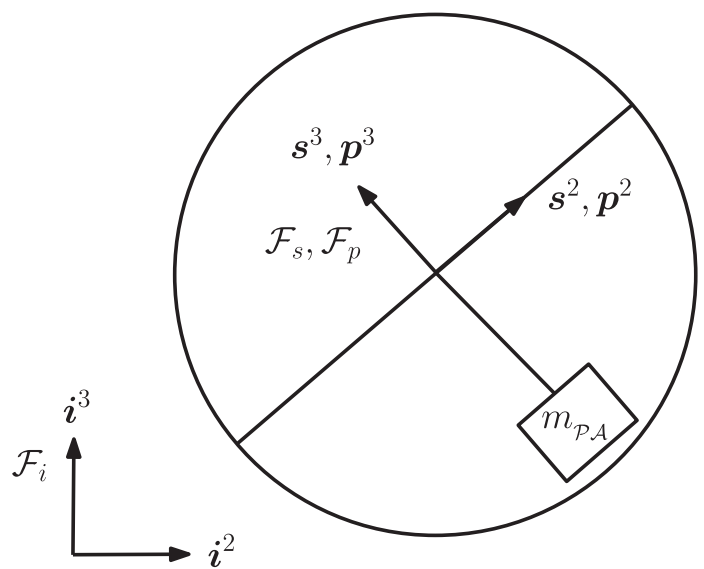

b) Front view

Fig. 4 Reference frames.

out in this section is envisioned to play a major role during the design phase of tumbleweed rovers by predicting the rolling stability behavior of designs with different mass distributions.

At this point, it is worth pondering why the results of [8] and [12] differ. Referring to Fig. 3a, the model developed in [12] assumed that the mass and inertia of the central rod was small and therefore negligible, whereas the rover built in [8] had a central rod made of steel with nonnegligible mass and inertia. The rolling instability documented in [8] is thought to stem from the inertia about the preferred axis being too small. To increase the inertia about the preferred axis, the addition of a spherical annulus, as shown in Fig. 3b, is proposed. The difficulty now lies in proving that the addition of a spherical annulus actually leads to a stable system. Increasing the inertia about the preferred axis seems logical given the majoraxis rule ([26] pp. 139-146). However, the major-axis rule is only applicable to a system modeled as a single quasi-rigid body, which the tumbleweed rover with its internal pendulum is most certainly not. As such, rolling stability of the tumbleweed rover will be assessed via Lyapunov's indirect method.

\section{A. Equilibrium State}

Before deriving the perturbed dynamics, it is necessary to determine the equilibrium state of the rover when it is subjected to external forces. When the rover is rotating nominally about the $s^{2}$ axis along a straight line, the direction cosine matrices $\mathbf{C}_{s i}$ and $\mathbf{C}_{p s}$ simplify to

$$
\mathbf{C}_{s i}^{*}=\mathbf{C}_{2}\left(\alpha^{*}\right) \quad \text { and } \quad \mathbf{C}_{p s}^{*}=\mathbf{C}_{2}\left(\phi^{*}\right)
$$

where $\alpha$ is the angle between $\mathcal{F}_{s}$ and $\mathcal{F}_{i}$, and $\phi$ is the angle between $\mathcal{F}_{p}$ and $\mathcal{F}_{s}$. At the equilibrium state, the pendulum will be tilted at a constant angle $\theta^{*}$ relative to the vertical axis because it is subject to torques created by the gravity field and by the power generation unit. This is depicted in Fig. 5. Note that the notation "*" is used to evaluate a term at the equilibrium state.

By the geometry of the problem, the angle $\theta$ is given by

$$
\theta=\alpha+\phi
$$

At the equilibrium state, when the pendulum is stationary relative to $\mathcal{F}_{i}$, it follows that

$$
\boldsymbol{\omega}_{s}^{s i *}=\omega^{*} \mathbf{1}_{2} \quad \text { and } \quad \boldsymbol{\omega}_{p}^{p s *}=-\omega^{*} \mathbf{1}_{2}
$$

where $\omega^{*}$ is the angular velocity of the rolling sphere at equilibrium about the $s^{2}$ axis. The expressions in Eq. (3) are obtained using Eq. ( $\underline{\mathrm{A} 1})$ with $\boldsymbol{\omega}_{p}^{p i *}=\mathbf{0}$. The reduced angular velocities can be written as

$$
\hat{\boldsymbol{\omega}}^{*}=\left[\begin{array}{ll}
\boldsymbol{\omega}_{s}^{s i * \top} & \omega_{p, 2}^{p s *}
\end{array}\right]^{\top}=\left[\begin{array}{ll}
\omega^{*} \mathbf{1}_{2}^{\top} & -\omega^{*}
\end{array}\right]^{\top}
$$

When the rover is rolling with a constant angular velocity, the term $\hat{\mathbf{M}}^{*} \dot{\hat{\boldsymbol{\omega}}}^{*}$ vanishes and Eq. (1) reduces to

$$
\hat{\boldsymbol{\tau}}^{\mathrm{non} *}=\underbrace{\Delta^{*} \mathbf{M}^{*} \dot{\boldsymbol{\Pi}}^{*} \hat{\boldsymbol{\omega}}^{*}}_{\mathbf{b}_{1}^{*}}+\underbrace{\Delta^{*} \nu^{*^{*}} \mathbf{M}^{*} \boldsymbol{\Pi}^{*} \hat{\boldsymbol{\omega}}^{*}}_{\mathbf{b}_{2}^{*}}+\underbrace{\Delta^{*} \mathbf{a}^{*}}_{\mathbf{b}_{3}^{*}}=\boldsymbol{\Delta}^{*} \boldsymbol{\tau}^{\mathrm{ext} *}
$$

At equilibrium, the term $\mathbf{b}_{1}^{*}$ reduces to

$$
\mathbf{b}_{1}^{*}=\boldsymbol{\Delta}^{*} \mathbf{M}^{*}\left[\begin{array}{c}
-r \omega^{* 2} \mathbf{1}_{3}^{\times} \mathbf{C}_{2}\left(\alpha^{*}\right)^{\top} \mathbf{1}_{2}^{\times} \mathbf{1}_{2} \\
\omega^{* 2} \mathbf{1}_{2}^{\times} \underbrace{\mathbf{\mathbf { C } _ { 2 }}\left(\phi^{*}\right) \mathbf{1}_{2}}_{\mathbf{1}_{2}}
\end{array}\right]=\mathbf{0}
$$

where $\dot{\boldsymbol{\Pi}}, \boldsymbol{\Delta}$, and $\mathbf{M}$ are given in Eqs. (A7-A9), respectively. Notice that the matrix multiplication of $\mathbf{C}_{2}(\phi)$ with $\overline{\mathbf{1}_{2}}$ reduces to $\mathbf{1}_{2}$ and that the matrix multiplication of the cross-product matrix of a column matrix by itself is $\mathbf{0}$ (i.e., $\mathbf{1}_{2}^{\times} \mathbf{1}_{2}=\mathbf{0}$ ). The term $\mathbf{b}_{2}^{*}$ can be written as

$$
\mathbf{b}_{2}^{*}=\boldsymbol{\Delta}^{*} \boldsymbol{\nu}^{*^{\times}} \mathbf{M}^{*} \boldsymbol{\nu}^{*}=\boldsymbol{\Delta}^{*}\left[\begin{array}{c}
\left(m_{\mathcal{S}}+m_{\mathcal{P}}\right) \dot{\mathbf{r}}_{i}^{s i *^{\times}} \dot{\mathbf{r}}_{i}^{s i *} \\
\omega^{* 2} \mathbf{1}_{2}^{\times} \mathbf{J}_{s}^{\mathcal{S}} \mathbf{1}_{2} \\
\boldsymbol{\omega}_{p}^{p i *^{\times}} \mathbf{c}_{p}^{\mathcal{P} p^{\times}} \mathbf{C}_{p i}^{*} \dot{\mathbf{r}}_{i}^{s i *}
\end{array}\right]=\mathbf{0}
$$

where $\nu=\Pi \hat{\boldsymbol{\omega}}$, as shown in Eq. (A6), $\nu^{\times}$is given in Eq. (A11), and $\boldsymbol{\omega}_{p}^{p i *}=\mathbf{0}$. In Eq. (ㄷ), observe that the terms $\dot{\mathbf{r}}_{i}^{s i \times} \dot{\mathbf{r}}_{i}^{s i}$ and $\overline{\mathbf{1}_{2}^{\times}} \mathbf{J}_{s}^{\mathcal{S}} \mathbf{1}_{2}$ reduce to $\mathbf{0}$. The term $\mathbf{b}_{3}^{*}$ can be written as

$$
\mathbf{b}_{3}^{*}=\boldsymbol{\Delta}^{*} \mathbf{a}^{*}=\left[\begin{array}{c}
-\mathbf{C}_{2}^{\top}\left(\alpha^{*}\right)\left(\mathbf{C}_{p i}^{*} \mathbf{1}_{3}\right)^{\times} \mathbf{c}_{p}^{\mathcal{P} p} g \\
-\mathbf{1}_{2}^{T}\left(\mathbf{C}_{p i}^{*} \mathbf{1}_{3}\right)^{\times} \mathbf{c}_{p}^{\mathcal{P} p} g
\end{array}\right]
$$

where $\mathbf{a}$ is given by Eq. (A10). Using the relation

$$
\mathbf{C}_{p i}^{*}=\mathbf{C}_{p s}^{*} \mathbf{C}_{s i}^{*}=\mathbf{C}_{2}\left(\phi^{*}+\alpha^{*}\right)=\mathbf{C}_{2}\left(\theta^{*}\right)
$$

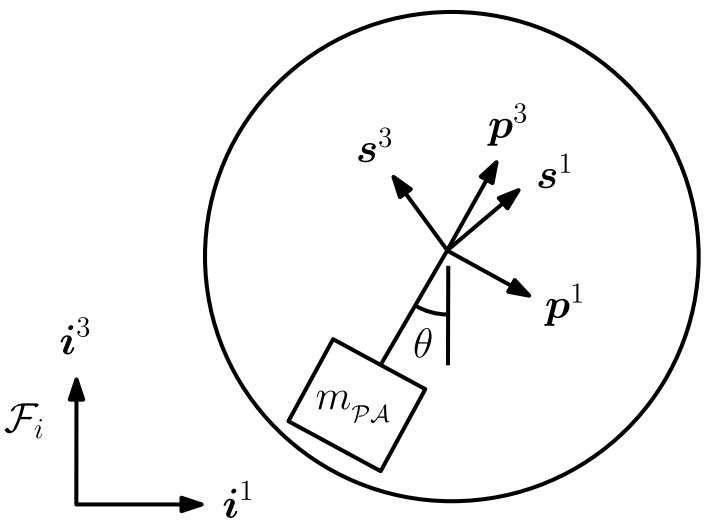

Fig. 5 Equilibrium position of payload. 
Equation (6) can be written as

$\mathbf{b}_{3}^{*}=\left[\begin{array}{c}0 \\ -g \mathbf{1}_{3}^{\top} \mathbf{c}_{p}^{\mathcal{P} p} \sin \theta^{*} \\ 0 \\ -g \mathbf{1}_{3}^{\top} \mathbf{c}_{p}^{\mathcal{P} p} \sin \theta^{*}\end{array}\right]=\left[\begin{array}{c}0 \\ g \ell_{\mathcal{P R}} \sin \theta^{*}\left(\frac{1}{2} m_{\mathcal{P R}}+m_{\mathcal{P A}}\right) \\ 0 \\ g \ell_{\mathcal{P R}} \sin \theta^{*}\left(\frac{1}{2} m_{\mathcal{P R}}+m_{\mathcal{P A}}\right)\end{array}\right]$

where the first moment of mass of the pendulum is $\mathbf{c}_{p}^{\mathcal{P} p}=$ $-\ell_{\mathcal{P R}}\left(\frac{1}{2} m_{\mathcal{P R}}+m_{\mathcal{P A}}\right) \mathbf{1}_{3}$. In this paper, the force that propels the rover forward will be modeled as an aerodynamic drag force defined as

$$
\mathbf{f}_{i}^{w}=\frac{1}{2} \rho A C_{d}\left\|\mathbf{v}_{i}^{w s}\right\|^{2} \frac{\mathbf{v}_{i}^{w s}}{\left\|\mathbf{v}_{i}^{w s}\right\|}
$$

where $\rho$ is the density of the surrounding air, $A$ is the frontal area of the rover, and $C_{d}$ is the drag coefficient. The term $\mathbf{v}_{i}^{w s}$ is the velocity of the wind relative to the sphere expressed in $\mathcal{F}_{i}$, referred to as the wind relative velocity, and is given by

$$
\mathbf{v}_{i}^{w s}=\mathbf{v}_{i}^{w i}-\mathbf{v}_{i}^{s i}
$$

where the term $\mathbf{v}_{i}^{w i}$ is the velocity of the wind relative to $\mathcal{F}_{i}$ expressed in $\mathcal{F}_{i}$, referred to as the wind absolute velocity, and where $\mathbf{v}_{i}^{s i}=\dot{\mathbf{r}}_{i}^{s i}$ is the translational velocity of the rover. A constant wind absolute velocity of $v_{i, 1}^{w i} \mathrm{~m} / \mathrm{s}$ acting in the $\boldsymbol{i}^{1}$ direction leads to an equilibrium aerodynamic force of

$$
\mathbf{f}_{i}^{w *}=\frac{1}{2} \rho A C_{d}\left(v_{i, 1}^{w i}-\omega^{*} r\right)^{2} \mathbf{1}_{1}
$$

The forces associated with translational damping $\mathbf{f}^{r}$, spin damping $\mathbf{t}^{s}$, and power generation $\mathbf{t}^{p}$ will also be included in the stability analysis, as seen in Fig. 6. The translational damping and spin damping terms are given by $[\underline{12}]$

$$
\mathbf{f}^{r}=\left[\begin{array}{c}
-b_{r} \dot{\mathbf{r}}_{i}^{s i} \\
\mathbf{0} \\
\mathbf{0}
\end{array}\right] \text { and } \mathbf{t}^{s}=\left[\begin{array}{c}
\mathbf{0} \\
-b_{s}\left(\mathbf{C}_{s i} \mathbf{1}_{3} \mathbf{1}_{3}^{\top} \mathbf{C}_{s i}^{\top} \boldsymbol{\omega}_{s}^{s i}\right) \\
\mathbf{0}
\end{array}\right]
$$

and the aerodynamic force and power generation terms are given by

$$
\mathbf{f}^{w}=\left[\begin{array}{c}
\mathbf{f}_{i}^{w} \\
\mathbf{0} \\
\mathbf{0}
\end{array}\right] \text { and } \mathbf{t}^{p}=\left[\begin{array}{c}
\mathbf{0} \\
b_{p} \omega_{p, 2}^{p s} \mathbf{C}_{p s}^{\top} \mathbf{1}_{2} \\
-b_{p} \omega_{p, 2}^{p s} \mathbf{1}_{2}
\end{array}\right]
$$

At equilibrium, the right-hand side term of Eq. (4) associated with the external forces simplifies to

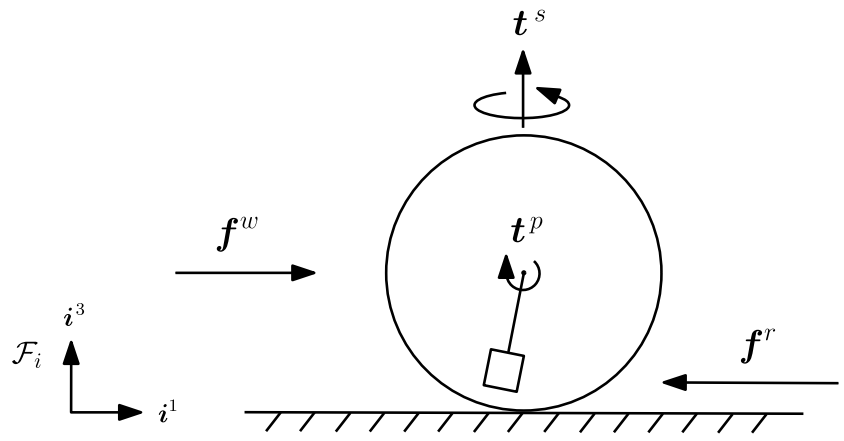

Fig. 6 External forces.

$$
\begin{aligned}
\boldsymbol{\Delta}^{*} \boldsymbol{\tau}^{\mathrm{ext} *} & =\boldsymbol{\Delta}^{*}\left(\mathbf{f}^{r *}+\mathbf{t}^{s *}+\mathbf{t}^{p *}+\mathbf{f}^{w *}\right) \\
& =\left[\begin{array}{c}
-r^{2} b_{r} \mathbf{1}_{2} \omega^{*}+\frac{1}{2} r \rho A C_{d}\left(v_{i, 1}^{w i}-r \omega^{*}\right)^{2} \mathbf{1}_{2} \\
b_{p} \omega^{*}
\end{array}\right]
\end{aligned}
$$

Combining the evaluated terms $\mathbf{b}_{1}^{*}, \mathbf{b}_{2}^{*}, \mathbf{b}_{3}^{*}$, and $\boldsymbol{\Delta}^{*} \boldsymbol{\tau}^{\mathrm{ext} *}$ in Eq. ( $\underline{4}$ ), it follows that

$$
\begin{aligned}
& {\left[\begin{array}{c}
0 \\
g \ell_{\mathcal{P R}} \sin \theta^{*}\left(\frac{1}{2} m_{\mathcal{P R}}+m_{\mathcal{P A}}\right) \\
0 \\
g \ell_{\mathcal{P R}} \sin \theta^{*}\left(\frac{1}{2} m_{\mathcal{P R}}+m_{\mathcal{P A}}\right)
\end{array}\right]} \\
& =\left[\begin{array}{c}
0 \\
-r^{2} b_{r} \omega^{*}+\frac{1}{2} r \rho A C_{d}\left(v_{i, 1}^{w i}-r \omega^{*}\right)^{2} \\
0 \\
b_{p} \omega^{*}
\end{array}\right]
\end{aligned}
$$

Subtracting the last row from the second row in Eq. (ㅇ) and solving the resulting quadratic equation for $\omega^{*}$, it follows that

$$
\omega^{*}=\frac{v_{i, 1}^{w i}}{r}+\frac{b_{r} r^{2}+b_{p}}{\rho A C_{d} r^{3}} \pm \frac{1}{r^{3}} \sqrt{\left[r^{2} v_{i, 1}^{w i}+\left(\frac{b_{r} r^{2}+b_{p}}{\rho A C_{d}}\right)\right]^{2}-r^{4} v_{i, 1}^{w i^{2}}}
$$

There are two possible solutions for $\omega^{*}$. The negative solution leads to a positive wind relative velocity, implying that the wind is pushing the rover forward. The positive solution leads to a negative wind relative velocity, implying that the wind acts in a way that resists the forward motion of the rover (i.e., acts as a resistive force). In this paper, the rolling stability of the equilibrium state is studied when the rover is being propelled forward by wind forces, therefore, only the negative solution is of interest. Isolating for $\theta^{*}$ in the last row of Eq. (8), the equilibrium angle of the pendulum can be written as a function of $\omega^{*}$ and the physical parameters of the rover as

$$
\theta^{*}=\sin ^{-1}\left(\frac{\omega^{*} b_{p}}{g \ell_{\mathcal{P R}}\left(\frac{1}{2} m_{\mathcal{P R}}+m_{\mathcal{P A}}\right)}\right)
$$

Notice that Eq. (10) corresponds to the sum of the moments acting on the pendulum at equilibrium taken about the center of the sphere and that Eq. (9) corresponds to the sum of the moments acting on the sphere at equilibrium taken about the center of the sphere.

\section{B. Perturbed Dynamics}

In this section, the perturbed dynamics of the tumbleweed rover about the equilibrium state will be derived and studied. The equations of motion of the tumbleweed rover given in Eq. (1) can also be written in terms of the augmented velocities $\nu$ as

$$
\mathbf{Y}^{\top} \overline{\mathbf{S}}^{\top}\left(\mathbf{M} \dot{\nu}+\nu^{\times} \mathbf{M} \nu+\mathbf{a}\right)=\mathbf{Y}^{\top} \overline{\mathbf{S}}^{\top} \boldsymbol{\tau}^{\mathrm{ext}}
$$

where the matrices $\mathbf{Y}$ and $\overline{\mathbf{S}}$ are given by Eqs. (A4) and (A5) in the Appendix. From Eq. (11), the equations of motion can be expressed in terms of the reduced coordinates $\dot{\hat{\mathbf{q}}}=\left[\begin{array}{ll}\dot{\boldsymbol{\theta}}^{s i \top} & \dot{\phi}\end{array}\right]^{\top}$ using the transformation

$$
\nu=\Psi \dot{\hat{\mathbf{q}}} \quad \text { and } \quad \dot{\nu}=\Psi \ddot{\hat{\mathbf{q}}}+\dot{\boldsymbol{\Psi}} \dot{\hat{\mathbf{q}}}
$$

where

$$
\boldsymbol{\Psi}=\overline{\mathbf{S}} \mathbf{Y}=\left[\begin{array}{cc}
-r \mathbf{1}_{3}^{\times} \mathbf{S}_{i}^{s i} & \mathbf{0} \\
\mathbf{S}_{i}^{s i} & \mathbf{0} \\
\mathbf{C}_{p s} \mathbf{S}_{i}^{s i} & \mathbf{1}_{2}
\end{array}\right]^{\top}
$$

Using a 3-1-2 Euler angle parameterization for $\mathbf{C}_{s i}$, that is, $\mathbf{C}_{s i}=\mathbf{C}_{2}(\alpha) \mathbf{C}_{1}(\beta) \mathbf{C}_{3}(\gamma)$, the matrix $\mathbf{S}_{s}^{s i}$ is given by ([26] pp. 26-28) 


$$
\mathbf{S}_{s}^{s i}=\left[\begin{array}{lll}
\mathbf{C}_{2}(\alpha) \mathbf{C}_{1}(\beta) \mathbf{1}_{3} & \mathbf{C}_{2}(\alpha) \mathbf{1}_{1} & \mathbf{1}_{2}
\end{array}\right]
$$

and the term $\mathbf{S}_{i}^{s i}$ is given by

$$
\mathbf{S}_{i}^{s i}=\mathbf{C}_{s i}^{\top} \mathbf{S}_{s}^{s i}=\left[\begin{array}{lll}
\mathbf{1}_{3} & \mathbf{C}_{3}^{\top}(\gamma) \mathbf{1}_{1} & \mathbf{C}_{3}^{\top}(\gamma) \mathbf{C}_{1}^{\top}(\beta) \mathbf{1}_{2}
\end{array}\right]
$$

The time derivative of $\boldsymbol{\Psi}$ is given by

$$
\dot{\boldsymbol{\Psi}}=\left[\begin{array}{cc}
-r \mathbf{1}_{3}^{\times} \dot{\mathbf{S}}_{i}^{s i} & \mathbf{0} \\
\dot{\mathbf{S}}_{s}^{s i} & \mathbf{0} \\
\dot{\mathbf{C}}_{p s} \mathbf{S}_{s}^{s i}+\mathbf{C}_{p s} \dot{\mathbf{S}}_{s}^{s i} & \mathbf{0}
\end{array}\right]
$$

where

$$
\dot{\mathbf{S}}_{s}^{s i}=\left[\begin{array}{lll}
\left(\dot{\mathbf{C}}_{2}(\alpha) \mathbf{C}_{1}(\beta)+\mathbf{C}_{2}(\alpha) \dot{\mathbf{C}}_{1}(\beta)\right) \mathbf{1}_{3} & \dot{\mathbf{C}}_{2}(\alpha) \mathbf{1}_{1} & \mathbf{0}
\end{array}\right]
$$

and

$$
\dot{\mathbf{S}}_{i}^{s i}=\left[\begin{array}{lll}
\mathbf{0} & \dot{\mathbf{C}}_{3}^{\top}(\gamma) \mathbf{1}_{1} & \left(\dot{\mathbf{C}}_{3}^{\top}(\gamma) \mathbf{C}_{1}^{\top}(\beta)+\mathbf{C}_{3}^{\top}(\gamma) \dot{\mathbf{C}}_{1}^{\top}(\beta)\right) \mathbf{1}_{2}
\end{array}\right]
$$

where the time derivatives of the direction cosine matrices are obtained using Poisson's equation:

$$
\begin{aligned}
\dot{\mathbf{C}}_{1}^{\top}(\beta) & =\dot{\beta} \mathbf{C}_{1}^{\top}(\beta) \mathbf{1}_{1}^{\times}, \\
\dot{\mathbf{C}}_{2}^{\top}(\alpha) & =\dot{\alpha} \mathbf{C}_{2}^{\top}(\alpha) \mathbf{1}_{2}^{\times} \quad \text { and } \quad \dot{\mathbf{C}}_{3}^{\top}(\gamma)=\dot{\gamma} \mathbf{C}_{3}^{\top}(\gamma) \mathbf{1}_{3}^{\times}
\end{aligned}
$$

Combining Eq. (11) and Eq. (12), the equations of motion in terms of the reduced coordinates can be written as

$$
\underbrace{\boldsymbol{\Psi}^{\top} \mathbf{M} \Psi}_{\overline{\mathbf{M}}} \ddot{\hat{\mathbf{q}}}=\underbrace{\Psi^{\top}\left(\tau^{\mathrm{ext}}-\mathbf{M} \dot{\boldsymbol{\hat { \mathbf { q } }}}-\nu^{\times} \mathbf{M \Psi} \dot{\hat{\mathbf{q}}}-\mathbf{a}\right)}_{\mathbf{f}(\hat{\mathbf{q}}, \dot{\hat{\mathbf{q}}})}
$$

To obtain the perturbed dynamics of the tumbleweed rover about the equilibrium state, the linearized equations of motions of the tumbleweed rover are obtained by performing a first-order Taylor series expansion on Eq. (13), resulting in

$$
\overline{\mathbf{M}}^{*} \delta \ddot{\hat{\mathbf{q}}}=\left.\frac{\partial \mathbf{f}}{\partial \hat{\mathbf{q}}}\right|_{\left(\hat{\mathbf{q}}^{*}, \dot{\hat{\mathbf{q}}}^{*}\right)} \delta \hat{\mathbf{q}}+\left.\frac{\partial \mathbf{f}}{\partial \dot{\hat{\mathbf{q}}}}\right|_{\left(\hat{\mathbf{q}}^{*}, \dot{\hat{\mathbf{q}}}^{*}\right)} \delta \dot{\hat{\mathbf{q}}}
$$

where the equilibrium states $\hat{\mathbf{q}}^{*}$ and $\dot{\hat{\mathbf{q}}}^{*}$ are given by

$$
\begin{aligned}
& \hat{\mathbf{q}}^{*}=\left[\begin{array}{c}
\boldsymbol{\theta}^{s i *} \\
\phi^{*}
\end{array}\right]=\left[\begin{array}{c}
\mathbf{1}_{2} \alpha^{*} \\
\phi^{*}
\end{array}\right] \text { and } \\
& \dot{\hat{\mathbf{q}}}^{*}=\left[\begin{array}{c}
\dot{\boldsymbol{\theta}}^{s i *} \\
\dot{\phi}^{*}
\end{array}\right]=\left[\begin{array}{c}
\mathbf{1}_{2} \dot{\alpha}^{*} \\
-\dot{\alpha}^{*}
\end{array}\right]=\left[\begin{array}{c}
\mathbf{1}_{2} \omega^{*} \\
-\omega^{*}
\end{array}\right]
\end{aligned}
$$

When evaluating $\dot{\hat{\mathbf{q}}}^{*}$, the term $\dot{\phi}^{*}$ is replaced with $-\dot{\alpha}^{*}$ following a time differentiation of Eq. (2). Because the sphere is rolling in the $s^{1}-s^{3}$ plane, the rate of change of the Euler angle $\alpha^{*}$ is $\dot{\alpha}^{*}$, which is equivalent to the angular velocity term $\omega^{*}$.

\section{Lyapunov's Indirect Method}

Equation (14) can be transformed into the first-order form

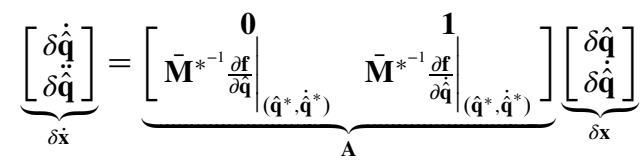

where the term $\delta \mathbf{x}$ is an augmented column matrix of the reduced coordinates and the rates of change of the reduced coordinates. The stability of the equilibrium state of the tumbleweed rover is studied using Lyapunov's indirect method ([27] pp. 356-359). The following well-known theorem establishes a relationship between the stability of the linearized system and the stability of the nonlinear system.

Theorem 4.1: Consider the linear approximation

$$
\delta \dot{\mathbf{x}}=\mathbf{A} \delta \mathbf{x}
$$

to the nonlinear system $\dot{\mathbf{x}}=\mathbf{f}(\mathbf{x})$, where

$$
\mathbf{A}=\left.\frac{\partial \mathbf{f}(\mathbf{x})}{\partial \mathbf{x}}\right|_{\mathbf{x}^{*}}
$$

and the eigenvalues of $\mathbf{A}$ are

$$
\lambda_{i}\{\mathbf{A}\}=\sigma_{i}+j \omega_{i}, \quad i=1, \ldots, n
$$

Let $\bar{\sigma}=\max _{i=1, \ldots, n}\left\{\sigma_{i}\right\}$. If the linear system is asymptotically stable (i.e., $\bar{\sigma}<0$ ), then the equilibrium point of the nonlinear system is locally asymptotically stable. If the linear system is unstable (i.e., $\bar{\sigma}>0$ ), then the equilibrium point of the nonlinear system is unstable. If all eigenvalues of $\mathbf{A}$ are in the left-half plane, but at least one is on the $j \omega$ axis, then one cannot conclude if the nonlinear system if stable or unstable.

To ensure that the tumbleweed rover is rolling stably about the equilibrium state, the real part of the eigenvalues of $\mathbf{A}$ must all be strictly negative.

\section{Numerical Results}

In this section, the nonlinear and perturbed equations of motion described earlier will be implemented in a MATLAB numerical simulation for validation. The second-order system given by Eq. (1) is transformed into state-space form as

$$
\left[\begin{array}{c}
\dot{\hat{\mathbf{q}}} \\
\dot{\hat{\boldsymbol{\omega}}}
\end{array}\right]=\left[\begin{array}{c}
\hat{\mathbf{S}}^{-1} \hat{\boldsymbol{\omega}} \\
\hat{\mathbf{M}}^{-1}\left(\hat{\boldsymbol{\tau}}^{\mathrm{ext}}-\hat{\boldsymbol{\tau}}^{\mathrm{non}}\right)
\end{array}\right]
$$

where

$$
\hat{\mathbf{S}}=\left[\begin{array}{cc}
\mathbf{S}_{s}^{s i} & 0 \\
\mathbf{0} & 1
\end{array}\right]
$$

Equations (15) and (16) are solved in MATLAB using the integrator ode 45 with an absolute and relative tolerance of $10^{-6}$. The simulations are performed in terrestrial conditions with the physical parameters of the tumbleweed rover given in Table 1 . The stability of the tumbleweed rover with and without the spherical annulus component will be studied using the linearized equations of motion and later validated through both linear and nonlinear simulations.

\section{A. Equilibrium State}

For a tumbleweed rover with the physical parameters given in Table 1 , the equilibrium angular velocity $\omega^{*}$, given by Eq. (9), is

$$
\begin{aligned}
\omega^{*} & =\frac{v_{i, 1}^{w i}}{r}+\frac{b_{r} r^{2}+b_{p}}{\rho A C_{d} r^{3}}-\frac{1}{r^{3}} \sqrt{\left[r^{2} v_{i, 1}^{w i}+\left(\frac{b_{r} r^{2}+b_{p}}{\rho A C_{d}}\right)\right]^{2}-r^{4} v_{1, i}^{w^{2}}} \\
& =2.627 \mathrm{rad} / \mathrm{s}
\end{aligned}
$$

and the pendulum equilibrium angle $\theta^{*}$, obtained using Eq. (10), is

$$
\theta^{*}=\sin ^{-1}\left(\frac{\omega^{*} b_{p}}{g \ell_{\mathcal{P R}}\left(\frac{1}{2} m_{\mathcal{P R}}+m_{\mathcal{P A}}\right)}\right)=0.0425 \mathrm{rad}=2.44 \mathrm{deg}
$$

A numerical simulation of the nonlinear system is performed in MATLAB with the initial conditions $\boldsymbol{\theta}^{s i}=\left[\begin{array}{lll}0 & 0 & 0\end{array}\right]^{\top} \operatorname{deg}, \boldsymbol{\omega}_{s}^{s i}=$ $\left[\begin{array}{lll}0 & 5 & 0\end{array}\right]^{\top} \mathrm{rad} / \mathrm{s}, \phi=0 \mathrm{deg}$, and $\dot{\phi}=0 \mathrm{rad} / \mathrm{s}$. Recall that the 
Table 1 Physical properties of the tumbleweed rover

\begin{tabular}{|c|c|c|}
\hline Property & Symbol & Value \\
\hline Drag coefficient of sphere & $C_{d}$ & 0.1 \\
\hline Mass of inflated sphere, $\mathrm{kg}$ & $m_{\mathcal{I S}}$ & 5 \\
\hline Mass of central rod, $\mathrm{kg}$ & $m_{\mathcal{C R}}$ & 5 \\
\hline Mass of spherical annulus, $\mathrm{kg}$ & $m_{\mathcal{S A}}$ & 8 \\
\hline Mass of pendulum rod, $\mathrm{kg}$ & $m_{\mathcal{P R}}$ & 1 \\
\hline Mass of payload, $\mathrm{kg}$ & $m_{\mathcal{P A}}$ & 10 \\
\hline Mass of pendulum, $\mathrm{kg}$ & $m_{\mathcal{P}}$ & $m_{\mathcal{P R}}+m_{\mathcal{P A}}$ \\
\hline Mass of sphere, $\mathrm{kg}$ & $m_{\mathcal{S}}$ & $m_{\mathcal{I S}}+m_{\mathcal{C R}}+m_{\mathcal{S A}}$ \\
\hline Length of pendulum rod, $\mathrm{m}$ & $\ell_{\mathcal{P R}}$ & 0.9 \\
\hline Length of central rod, $\mathrm{m}$ & $\ell_{\mathcal{C R}}$ & 1 \\
\hline Width of pendulum rod, $\mathrm{m}$ & $w_{\mathcal{P R}}$ & 0.025 \\
\hline Width of central rod, $\mathrm{m}$ & $w_{\mathcal{C R}}$ & 0.025 \\
\hline Width of spherical annulus, $\mathrm{m}$ & $w_{\mathcal{S A}}$ & 0.2 \\
\hline Height of payload, m & $h_{\mathcal{P}}$ & 0.1 \\
\hline Radius of inflated sphere, $\mathrm{m}$ & $r$ & 1 \\
\hline Sphere rolling damping coefficient, $\mathrm{kg} \cdot \mathrm{m}^{2} / \mathrm{s}$ & $b_{r}$ & 3.5 \\
\hline Sphere spin damping coefficient, $\mathrm{kg} \cdot \mathrm{m}^{2} / \mathrm{s}$ & $b_{s}$ & $r^{2} b_{r}$ \\
\hline Pendulum damping coefficient, $\mathrm{kg} \cdot \mathrm{m}^{2} / \mathrm{s}$ & $b_{p}$ & 1.5 \\
\hline Wind absolute velocity, $\mathrm{m} / \mathrm{s}$ & $\mathbf{v}_{\dot{b}}^{w i}$ & $10.7 \mathbf{1}_{1}$ \\
\hline First moment of mass of pendulum, $\mathrm{kg} \cdot \mathrm{m}$ & $c_{p}^{p p}$ & $-\ell_{\mathcal{P R}}\left(\frac{1}{2} m_{\mathcal{P R}}+m_{\mathcal{P A}}\right) \mathbf{1}_{3}$ \\
\hline Second moment of mass of inflated sphere, $\mathrm{kg} \cdot \mathrm{m}^{2}$ & $\mathbf{J}_{S}^{\mathcal{I} S S}$ & $\frac{2}{3} m_{\mathcal{I S}} r^{3} \mathbf{1}$ \\
\hline Second moment of mass of spherical annulus, $\mathrm{kg} \cdot \mathrm{m}^{2}$ & $\mathbf{J}_{s}^{\mathcal{S} \mathcal{A} s}$ & $\operatorname{diag}\left(\frac{1}{6} m_{\mathcal{S A}}\left(3 r^{2}+2 h_{\mathcal{S} \mathcal{A}}^{2}\right), m_{\mathcal{S A}} r^{2},\left(\frac{1}{6} m_{\mathcal{S A}}\left(3 r^{2}+2 h_{\mathcal{S} \mathcal{A}}^{2}\right)\right)\right.$ \\
\hline Second moment of mass of central rod, $\mathrm{kg} \cdot \mathrm{m}^{2}$ & $\mathbf{J}_{s}^{\mathcal{C R} s}$ & $\operatorname{diag}\left(\frac{1}{12} m_{\mathcal{C R}}\left(\ell_{\mathcal{C R}}^{2}+w_{\mathcal{C R}}^{2}\right), \frac{1}{6} m_{\mathcal{C R}} w_{\mathcal{C R}}^{2}, \frac{1}{12} m_{\mathcal{C R}}\left(\ell_{\mathcal{C R}}^{2}+w_{\mathcal{C R}}^{2}\right)\right)$ \\
\hline Second moment of mass of pendulum $\mathrm{rod}, \mathrm{kg} \cdot \mathrm{m}^{2}$ & $\mathbf{J}_{p}^{\mathcal{P R} p}$ & $\operatorname{diag}\left(\frac{1}{12} m_{\mathcal{P R}}\left(4 \ell_{\mathcal{P R}}+w_{\mathcal{P R}}^{2}\right), \frac{1}{12} m_{\mathcal{P R}}\left(4 \ell_{\mathcal{P} \mathcal{R}}^{2}+w_{\mathcal{P} \mathcal{R}}^{2}\right), \frac{1}{6} m_{\mathcal{P R}} w_{\mathcal{P} \mathcal{R}}^{2}\right)$ \\
\hline Second moment of mass of payload, $\mathrm{kg} \cdot \mathrm{m}^{2}$ & $\mathbf{J}_{p}^{\mathcal{P} \mathcal{A}_{p}}$ & $\operatorname{diag}\left(\frac{1}{6} m_{\mathcal{P A}}\left(h_{\mathcal{P}_{\mathcal{A}}}^{2}+6 \ell_{\mathcal{P}_{\mathcal{A}}}^{2}\right), \frac{1}{6} m_{\mathcal{P} \mathcal{A}}\left(h_{\mathcal{P}_{\mathcal{A} 2}}^{2}+6 \ell_{\mathcal{P}_{\mathcal{A}}}^{2}\right), \frac{1}{6} m_{\mathcal{P}_{\mathcal{A}}} h_{\mathcal{P}_{\mathcal{A}}}^{2}\right)$ \\
\hline Second moment of mass of pendulum, $\mathrm{kg} \cdot \mathrm{m}^{2}$ & $\mathbf{J}_{p}^{\mathcal{P} p}$ & $\mathbf{J}_{p}^{\mathcal{P R} p}+\mathbf{J}_{p}^{\mathcal{P} \mathcal{A} p}$ \\
\hline Second moment of mass of sphere, $\mathrm{kg} \cdot \mathrm{m}^{2}$ & $\mathbf{J}_{s}^{\mathcal{S} s}$ & $\mathbf{J}_{s}^{\mathcal{I S} s}+\mathbf{J}_{s}^{\mathcal{C R} s}+\mathbf{J}_{s}^{\mathcal{S A} s}$ \\
\hline
\end{tabular}

direction cosine matrix $\mathbf{C}_{s i}$ is parameterized by the Euler sequence $\mathbf{C}_{s i}=\mathbf{C}_{2}(\alpha) \mathbf{C}_{1}(\beta) \mathbf{C}_{3}(\gamma)$ and that $\boldsymbol{\theta}^{s i}=\left[\begin{array}{lll}\gamma & \alpha & \beta\end{array}\right]^{\top}$. Figure 7 shows that only the angles $\alpha$ and $\phi$ change with time because the rover is rolling nominally about the $s^{2}$ axis. It takes the rover approximately 30 s for the states $\omega_{s, 2}^{s i}$ and $\dot{\phi}$ to converge to the calculated equilibrium values of $\omega_{s, 2}^{s i}=\omega^{*}=2.63$ and $\dot{\phi}=-\omega^{*}=-2.63 \mathrm{rad} / \mathrm{s}$, thus numerically validating Eqs. (9) and (10). In Fig. 8, the pendulum angle $\theta$ is obtained by the addition of $\alpha$ and $\phi$. As time increases, the angle $\theta$ converges to the calculated equilibrium angle of $\theta^{*}=$ $2.44 \mathrm{deg}$.
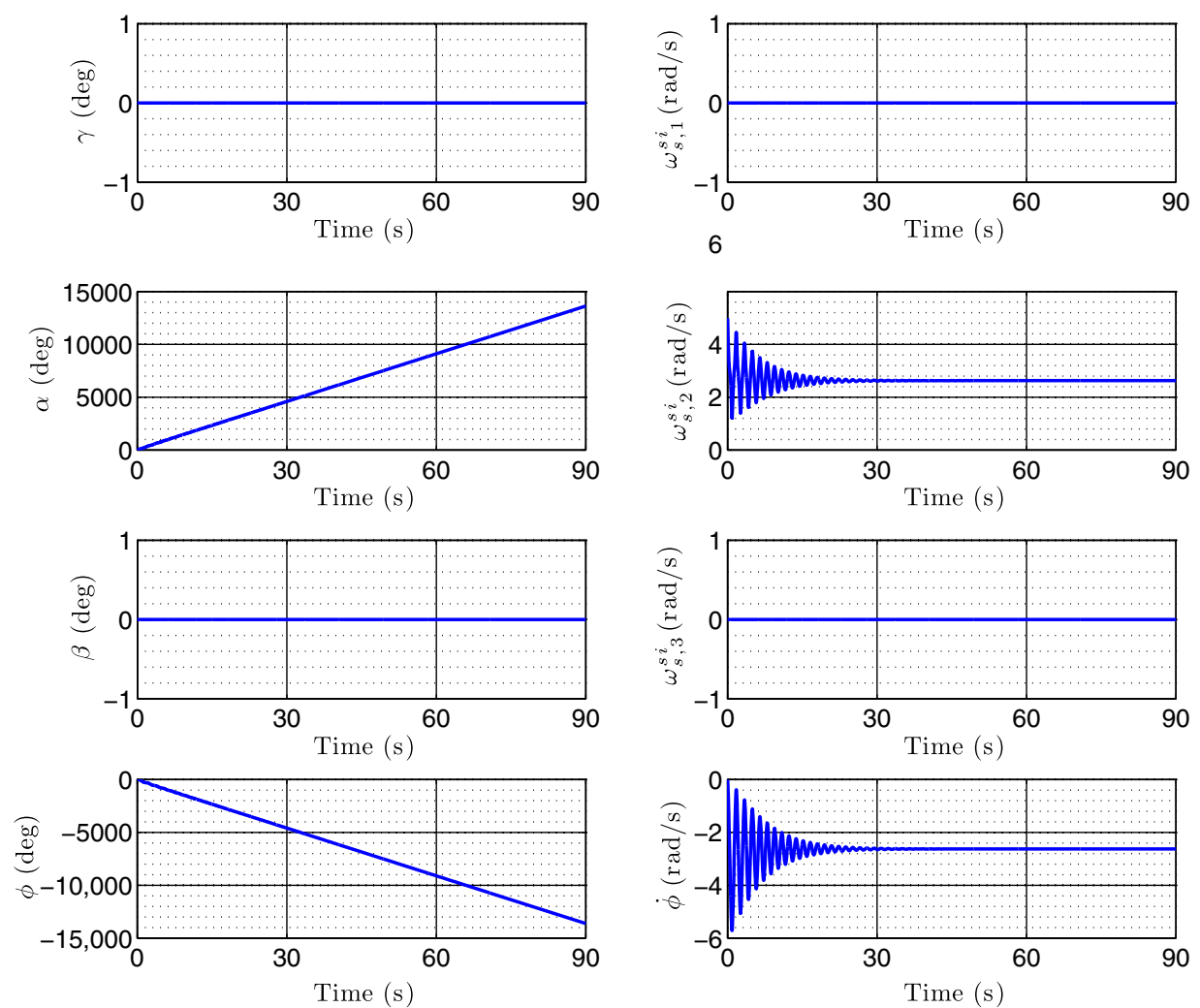

Fig. 7 Equilibrium state of the tumbleweed rover. 

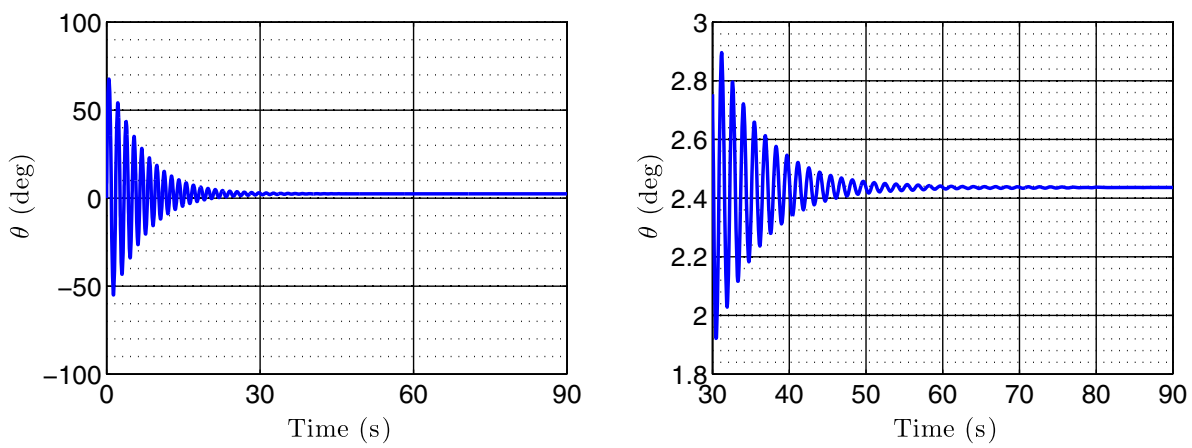

Fig. 8 Pendulum angle at equilibrium state.
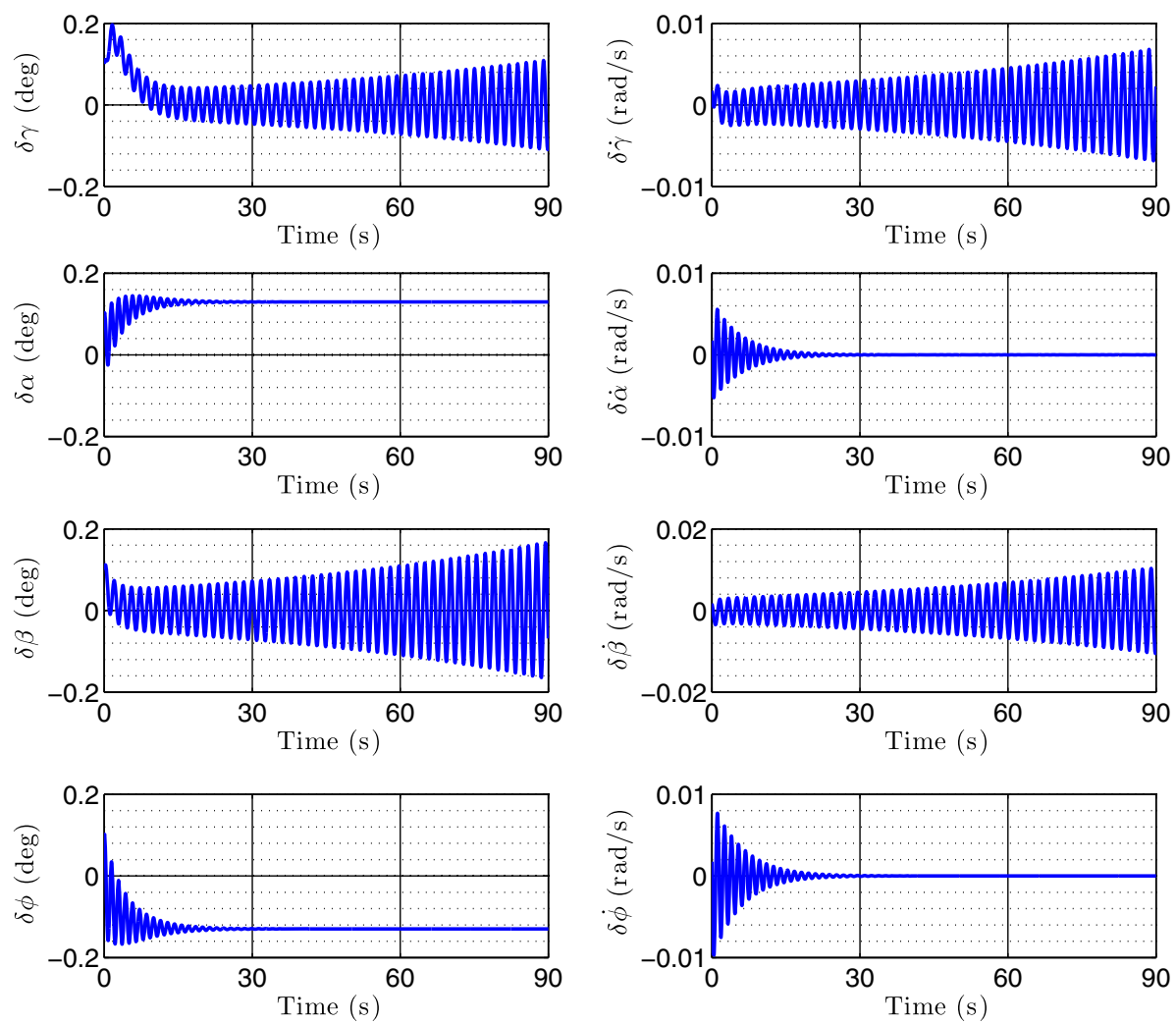

Fig. 9 Response from initial conditions of the perturbed dynamics without the spherical annulus component.

\section{B. Rover Instability}

The eigenvalues of $\mathbf{A}$, given in Eq. (15), are

$$
\begin{aligned}
& \boldsymbol{\Lambda}=\operatorname{diag}(0.0152+3.5929 j, 0.0152-3.5929 j,-0.7601,-0.3396 \\
& -0.3029,-0.1669+4.4222 j,-0.1669-4.4222 j, 0)
\end{aligned}
$$

where $\boldsymbol{\Lambda}$ is a diagonal matrix containing the eigenvalues of $\mathbf{A}$. The Jacobians in Eq. (15) are computed using the symbolic toolbox in MATLAB due to the complex nature of the $\mathbf{f}(\hat{\mathbf{q}}, \dot{\hat{\mathbf{q}}})$ term. When evaluating the terms $\overline{\mathbf{M}}^{*}$ and

$$
\left.\frac{\partial \mathbf{f}}{\partial \dot{\hat{\mathbf{q}}}}\right|_{\left(\hat{\mathbf{q}}^{*}, \dot{\hat{\mathbf{q}}}^{*}\right)}
$$

it is necessary to substitute the addition $\left(\alpha^{*}+\phi^{*}\right)$ with the term $\theta^{*}$, given by Eq. (10). The matrix $\boldsymbol{\Lambda}$ contains eigenvalues located in the open right-half plane, and by Theorem 4.1, the equilibrium state of the nonlinear system is unstable.

The response of the perturbed dynamics described by Eq. (14) [or equivalently given by Eq. (15)] from a set of initial conditions is shown in Fig. 9. Notice that the initial perturbations associated with the states $\delta \gamma, \delta \dot{\gamma}, \delta \beta$, and $\delta \dot{\beta}$ are amplified as time increases, indicating that the rover is unstable.

In Fig. 10, the nonlinear equations of motion of the tumbleweed rover are perturbed about the equilibrium state to verify that the tumbleweed rover behaves according to the predictions made using Lyapunov's indirect method. The initial angular velocities used are $\boldsymbol{\omega}_{s}^{s i}=\left[\begin{array}{lll}0.1 & 2.8 & 0.1\end{array}\right]^{T}$ and $\dot{\phi}=-2.8 \mathrm{rad} / \mathrm{s}$, and the initial Euler angles are $\boldsymbol{\theta}^{s i}=\left[\begin{array}{lll}0.1 & 0.1 & 0.1\end{array}\right]^{\top}$ and $\phi=0.1 \mathrm{deg}$. In Fig. 10, the perturbations associated with the states $\gamma$ and $\beta$ increase with time, causing the states $\omega_{s, 2}^{s i}$ and $\dot{\phi}$ to start to shift away from the equilibrium configuration at $50 \mathrm{~s}$. Note that, in reality, the tumbleweed rover will be exposed to much larger perturbations and can be expected to diverge from the equilibrium configuration much faster. The results obtained in field tests agree well with these predictions, where the tumbleweed rover rapidly transitioned from rolling about the preferred axis to an unstable configuration [8].

\section{Rover Stability}

In this section, the rolling stability of a tumbleweed rover that integrates a spherical annulus component, as seen in Fig. 3b, is studied. For the tumbleweed rover with a spherical annulus, the term $\mathbf{A}$ of Eq. (15) can be written as 

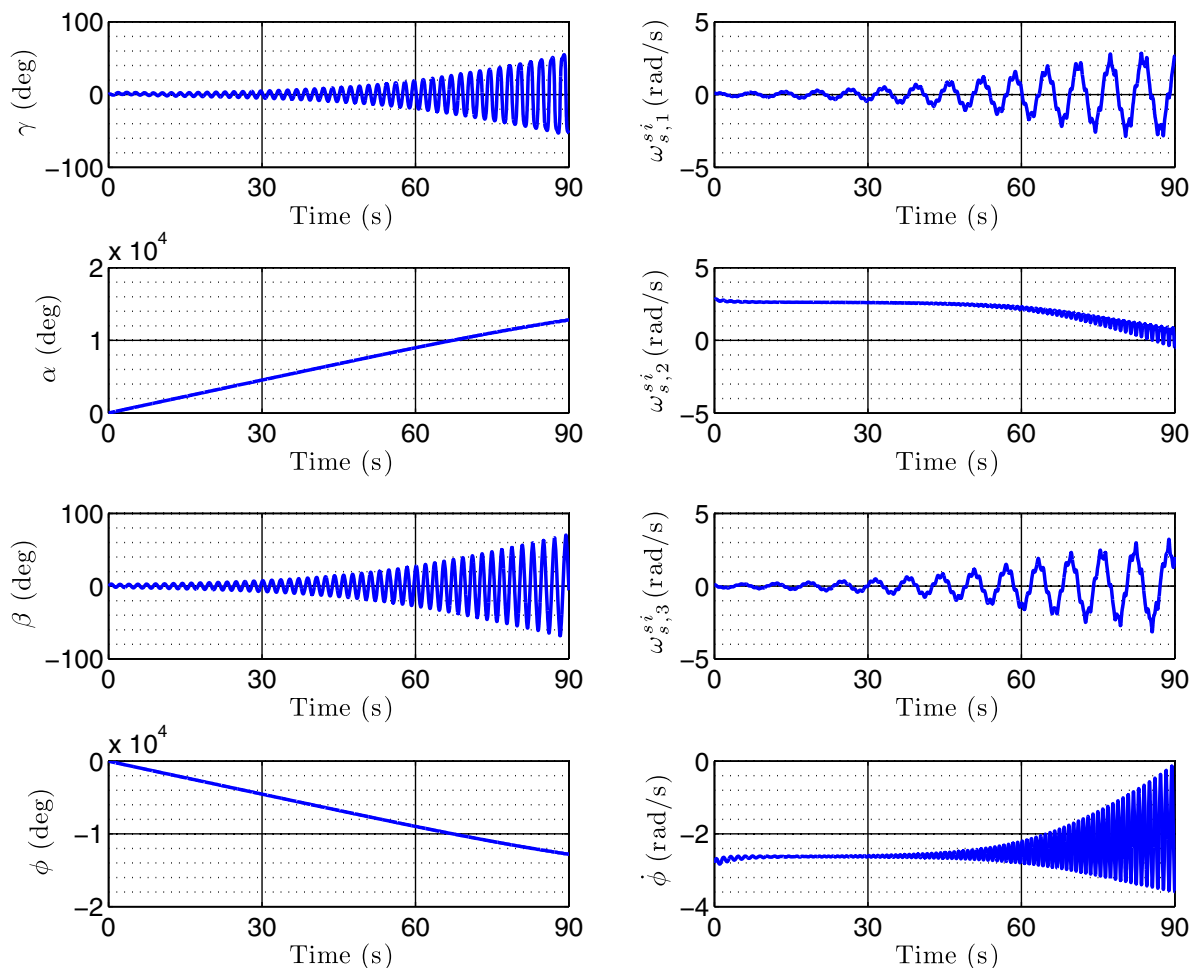

Fig. 10 Nonlinear system response without the spherical annulus component.
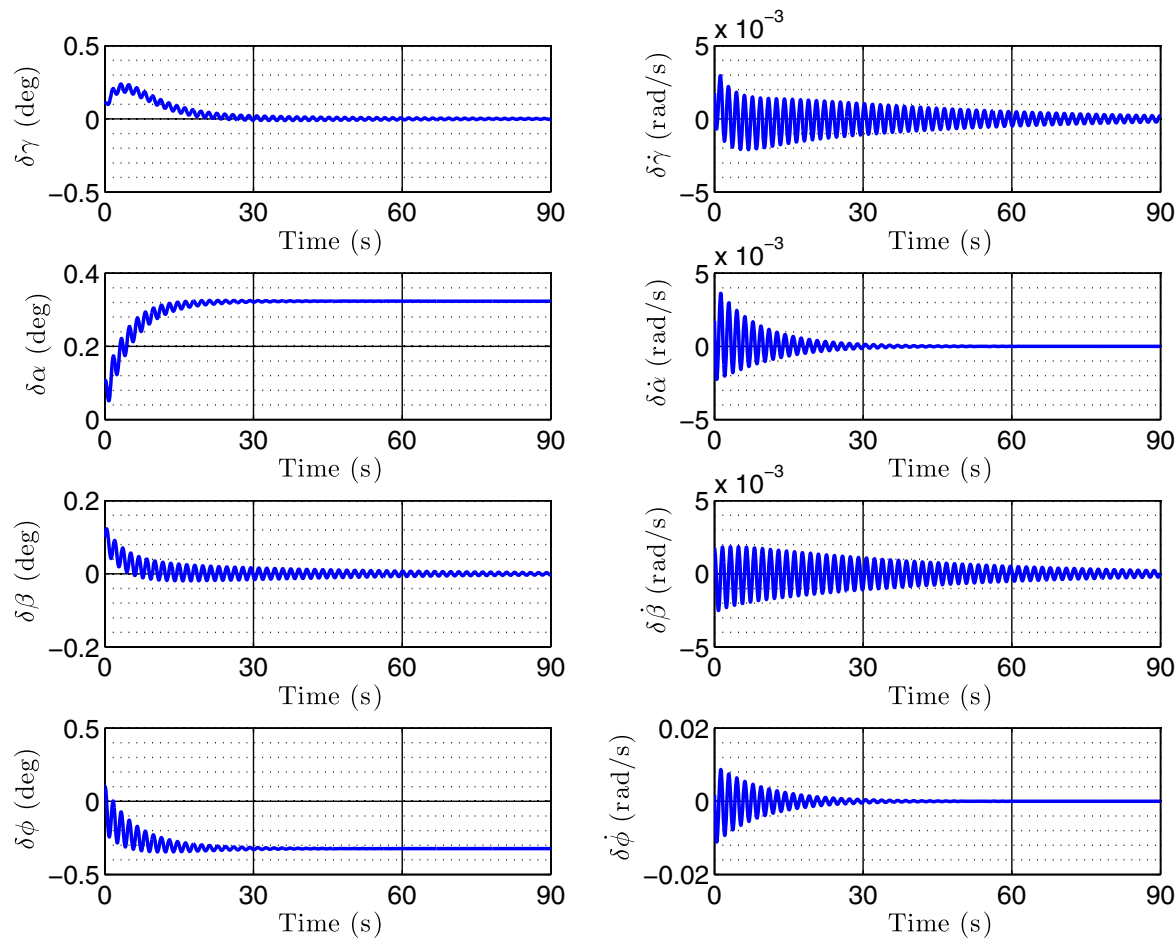

Fig. 11 Response from initial conditions of the perturbed dynamics with the spherical annulus component.

$$
\mathbf{A}=\left[\begin{array}{cccccccc}
0 & 0 & 0 & 0 & 1.0000 & 0 & 0 & 0 \\
0 & 0 & 0 & 0 & 0 & 1.0000 & 0 & 0 \\
0 & 0 & 0 & 0 & 0 & 0 & 1.0000 & 0 \\
0 & 0 & 0 & 0 & 0 & 0 & 0 & 1.0000 \\
-0.0039 & -1.1449 & 0 & 0 & -0.3280 & -3.7730 & 0 & 0 \\
0.6608 & -3.5136 & 0 & 0 & 3.0809 & -0.1880 & 0 & 0 \\
0 & 0 & -3.5101 & -3.5101 & 0 & 0 & -0.2274 & -0.0064 \\
0 & 0 & -11.4852 & -11.4852 & 0 & 0 & -0.0286 & -0.1797
\end{array}\right]
$$



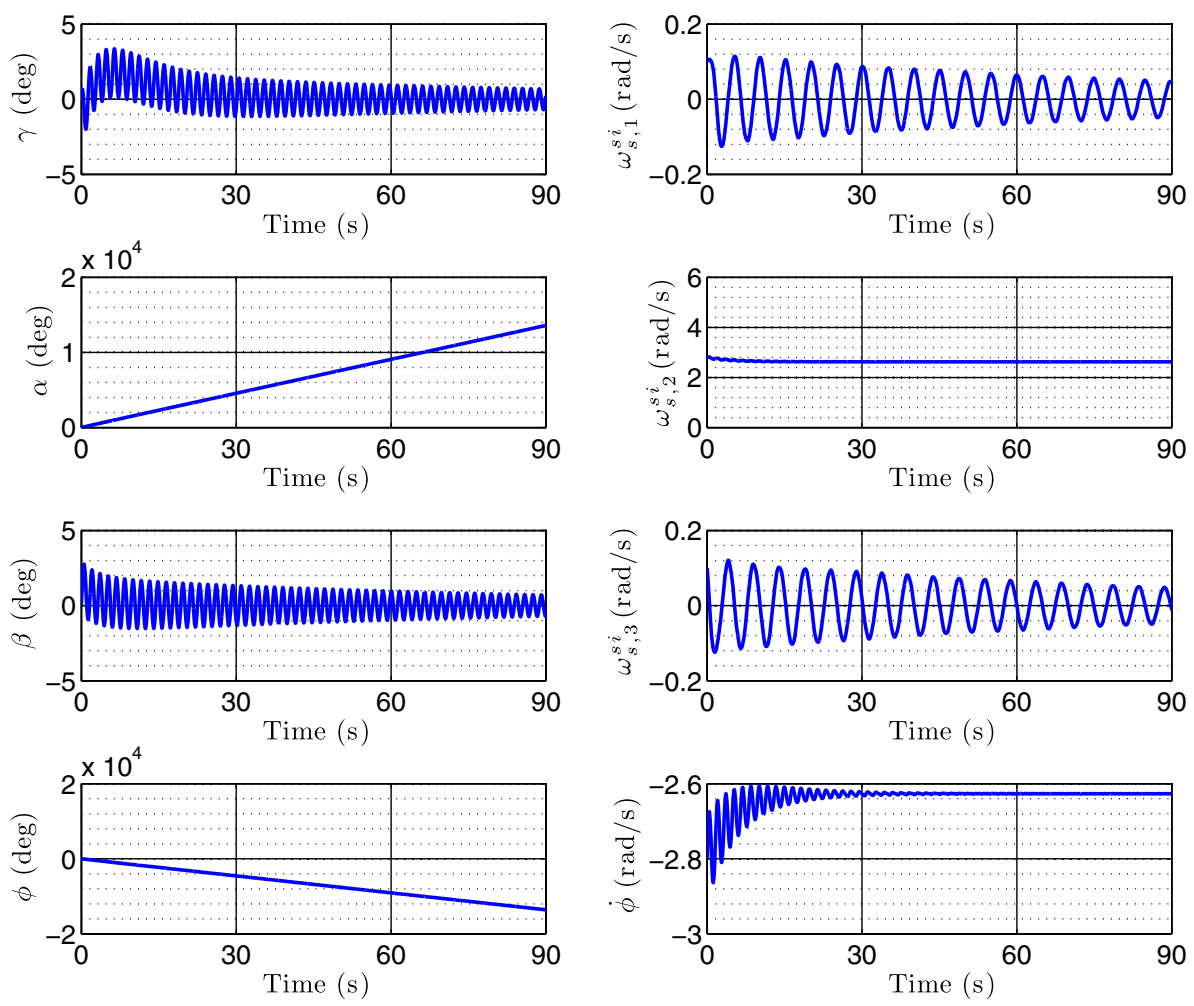

Fig. 12 Nonlinear system response with the spherical annulus component.

$$
\Lambda=\operatorname{diag}(-0.1012+3.8710 j,-0.1012-3.8710 j,-0.3094,-0.2047, \quad-0.1645,-0.0210+3.8900 j,-0.0210-3.8900 j, 0)
$$

where $\boldsymbol{\Lambda}$ is a diagonal matrix containing the eigenvalues of $\mathbf{A}$. Here, the matrix $\boldsymbol{\Lambda}$ contains an eigenvalue located on the imaginary axis and, by Theorem 4.1, the stability analysis of the nonlinear system is inconclusive. However, notice from Eqs. (15) and (17) that $\delta \alpha, \delta \dot{\alpha}$, $\delta \phi$, and $\delta \dot{\phi}$ can be decoupled from $\delta \gamma, \delta \dot{\gamma}, \overline{\delta \beta}$, and $\delta \bar{\beta}$. As such, it is possible to eliminate the rows and columns associated with these states and write a reduced form of Eq. (15):

$$
\delta \dot{\hat{\mathbf{x}}}=\hat{\mathbf{A}} \delta \hat{\mathbf{x}}
$$

where

$$
\delta \hat{\mathbf{x}}=\left[\begin{array}{llll}
\delta \gamma & \delta \beta & \delta \dot{\gamma} & \delta \dot{\beta}
\end{array}\right]^{\top}
$$

and

$$
\hat{\mathbf{A}}=\left[\begin{array}{cccc}
0 & 0 & 1.0000 & 0 \\
0 & 0 & 0 & 1.0000 \\
-0.0039 & -1.1449 & -0.3280 & -3.7730 \\
0.6608 & -3.5136 & 3.0809 & -0.1880
\end{array}\right]
$$

The states $\delta \alpha$ and $\delta \phi$ that are eliminated are associated with the forward and backward motion of the sphere and the pendulum, and they are of no interest when studying the rolling stability of the tumbleweed rover. For the rover to roll stably about the $s^{2}$ axis, only the states $\delta \gamma, \delta \dot{\gamma}, \delta \beta$, and $\delta \dot{\beta}$, associated with the yaw and the roll of the sphere, must converge to zero. A similar approach is used to assess stability of a rolling coin in [28]. Therefore, the analysis of the eigenvalues of $\hat{\mathbf{A}}$ is sufficient to conclude on the rolling stability of the rover. The eigenvalues of $\hat{\mathbf{A}}$ are

$$
\begin{aligned}
\hat{\boldsymbol{\Lambda}}= & \operatorname{diag}(-0.0210+3.8900 j,-0.0210 \\
& -3.8900 j,-0.3094,-0.1645)
\end{aligned}
$$

where $\hat{\boldsymbol{\Lambda}}$ is a diagonal matrix containing the eigenvalues of $\hat{\mathbf{A}}$. The equilibrium state of the reduced system is said to be locally asymptotically stable by Theorem 4.1 because all of the eigenvalues lie in the open left-half complex plane.

In Fig. 11, the perturbations associated with the states $\delta \gamma, \delta \dot{\gamma}, \delta \beta$, and $\dot{\delta \beta}$ decay to zero with time, confirming that, when the tumbleweed rover is equipped with the spherical annulus, the equilibrium state of the reduced system is locally asymptotically stable.

To validate the conclusion drawn based on the linearized system, the nonlinear system with the spherical annulus component is simulated with initial angular velocities of $\boldsymbol{\omega}_{s}^{s i}=\left[\begin{array}{lll}0.1 & 2.8 & 0.1\end{array}\right]^{\top}$ and $\dot{\phi}=-2.8 \mathrm{rad} / \mathrm{s}$ and the initial Euler angles of $\boldsymbol{\theta}^{\text {si }}=$ $\left[\begin{array}{lll}0.1 & 0.1 & 0.1\end{array}\right]^{T}$ and $\phi=0.1 \mathrm{deg}$. The nonlinear simulation, shown in Fig. 12, converges to the equilibrium state as predicted by Lyapunov's indirect method. The addition of the spherical annulus leads to a system that is stable about the equilibrium state.

To ensure that the results are not sensitive to the physical parameters used in the numerical simulations, the mass of the spherical annulus was increased and decreased by $10 \%$, yielding unchanged stability conclusions.

\section{Conclusions}

In this paper, a procedure to analyze the rolling stability of a powergenerating tumbleweed rover was formulated and later validated through numerical simulations. Using Lyapunov's indirect method, it was rigorously shown that the mass needs to be distributed in a particular way for the tumbleweed rover to be dynamically stable about the $s^{2}$ axis. It was concluded that a rover with a spherical shell with a uniform mass distribution leads to rolling instabilities. Moreover, the addition of external mass about the preferred axis of rotation leads to stable rolling of the rover and is a simple solution to the stability issues raised in [8]. An important observation made in this study was that the use of Euler angles greatly facilitated the linearization of the equations of motion of the tumbleweed rover by decoupling the dynamics of the states of interest from the overall system. If the equations of motion had been linearized using angular velocities instead of Euler angle rates, it is not clear how Lyapunov's indirect 
method would be applied. The methods described in this paper to investigate the dynamic stability of equilibrium configurations can be applied to a wide variety of spherical robots with different mass distributions.

\section{Appendix A: Derivation of Equations of Motion}

The material presented in this Appendix is a brief review of the work presented in [12]. This review is necessary to properly derive the perturbed dynamics of the tumbleweed rover, as is done in Sec. IV. This Appendix introduces the generalized and the reduced coordinates of the system, the kinematic system constraints, and the expressions for the kinetic energy and the potential energy of the system. Also, several useful kinematic relations are defined that greatly simplify the derivation.

The generalized coordinates used to describe the motion of the rover are

$$
\mathbf{q}=\left[\begin{array}{lll}
\mathbf{r}_{i}^{s i \top} & \boldsymbol{\theta}^{s i \top} & \boldsymbol{\theta}^{p i \top}
\end{array}\right]^{\top}
$$

where the term $\mathbf{r}_{i}^{s i}$ relates the position of the origin of $\mathcal{F}_{s}$ relative to the origin of $\mathcal{F}_{i}$ expressed in the inertial frame $\mathcal{F}_{i}$. The angular velocities of the system are given by

$$
\boldsymbol{\omega}_{s}^{s i}=\mathbf{S}_{s}^{s i} \dot{\boldsymbol{\theta}}^{s i} \quad \text { and } \quad \boldsymbol{\omega}_{p}^{p i}=\mathbf{S}_{p}^{p i} \dot{\boldsymbol{\theta}}^{p i}
$$

where $\mathbf{S}_{s}^{s i}$ and $\mathbf{S}_{p}^{p i}$ are the mapping matrices between the Euler angle rates and the angular velocities ([26] pp. 26-28). Here, the term $\boldsymbol{\omega}_{s}^{s i}$ describes the angular velocity of $\overline{\mathcal{F}}_{s}$ relative to $\mathcal{F}_{i}$ expressed in $\mathcal{F}_{s}$, and the term $\omega_{p}^{p l}$ describes the angular velocity of $\mathcal{F}_{p}$ relative to $\mathcal{F}_{i}$ expressed in $\mathcal{F}_{p}$. The angular velocity of $\boldsymbol{\omega}_{p}^{p i}$ can also be expressed as

$$
\boldsymbol{\omega}_{p}^{p i}=\boldsymbol{\omega}_{p}^{p s}+\mathbf{C}_{p s} \boldsymbol{\omega}_{s}^{s i}
$$

where $\boldsymbol{\omega}_{p}^{p s}$ describes the angular velocity of $\mathcal{F}_{p}$ relative to $\mathcal{F}_{s}$ expressed in $\mathcal{F}_{p}$ and $\mathbf{C}_{p s}=\mathbf{C}_{p i} \mathbf{C}_{s i}^{\top}$. When studying the dynamics of the tumbleweed rover, two kinematic constraints are present: the noslip rolling constraint and the pendulum and central rod constraint. The no-slip criterion for the rolling sphere can be expressed as

$$
\dot{\mathbf{r}}_{i}^{s i}=-r \mathbf{1}_{3}^{\times} \mathbf{C}_{s i} \boldsymbol{\omega}_{s}^{s i}=-r \mathbf{1}_{3}^{\times} \mathbf{C}_{s i}^{\top} \mathbf{S}_{s}^{s i} \dot{\boldsymbol{\theta}}^{s i}
$$

Because the pendulum is constrained to rotate about the $s^{2}$ axis, Eq. (A1) reduces to

$$
\boldsymbol{\omega}_{p}^{p i}=\mathbf{1}_{2} \dot{\phi}+\mathbf{C}_{p s} \boldsymbol{\omega}_{s}^{s i}
$$

using $\boldsymbol{\omega}_{p}^{p s}=\mathbf{1}_{2} \dot{\phi}$, where the angle $\phi$ describes the orientation between $\mathcal{F}_{p}$ and $\mathcal{F}_{s}$. Because the pendulum is rotating about the $\boldsymbol{s}^{2}$ axis, the direction cosine matrix describing the orientation of $\mathcal{F}_{p}$ relative to $\mathcal{F}_{s}$ is $\mathbf{C}_{p s}=\mathbf{C}_{2}(\phi)$, where the direction cosine matrix $\mathbf{C}_{2}$ is associated with a principal rotation about the 2 axis ([26] p. 15). In terms of the Euler angle rates, the preceding can be written as

$$
\dot{\boldsymbol{\theta}}^{p i}=\mathbf{S}_{p}^{p i-1} \mathbf{1}_{2} \dot{\boldsymbol{\phi}}+\mathbf{S}_{p}^{p i-1} \mathbf{C}_{p s} \dot{\boldsymbol{\theta}}^{s i}
$$

where it is assumed that singularities associated with $\mathbf{S}_{p}^{p i}$ are avoided. Equations (A2) and (A3) can be combined in a matrix form as

$$
\underbrace{\left[\begin{array}{c}
\dot{\mathbf{r}}_{i}^{s i} \\
\dot{\boldsymbol{\theta}}^{s i} \\
\dot{\boldsymbol{\theta}}^{p i}
\end{array}\right]}_{\dot{\mathbf{q}}}=\underbrace{\left[\begin{array}{cc}
-r \mathbf{1}_{3}^{\times} \mathbf{C}_{s i}^{\top} \mathbf{S}_{s}^{s i} & \mathbf{0} \\
\mathbf{1} & \mathbf{0} \\
\mathbf{S}_{p}^{p i-1} \mathbf{C}_{p s} \mathbf{S}_{s}^{s i} & \mathbf{S}_{p}^{p i-1} \mathbf{1}_{2}
\end{array}\right]}_{\mathbf{r}} \underbrace{\left[\begin{array}{c}
\dot{\boldsymbol{\theta}}^{s i} \\
\dot{\boldsymbol{\phi}}
\end{array}\right]}_{\dot{\hat{\mathbf{q}}}}
$$

where the matrix $\mathbf{Y}$ is the augmented constraint matrix mapping the reduced coordinates $\dot{\hat{\mathbf{q}}}$ to the dependent generalized coordinates $\dot{\mathbf{q}}$. In an effort to keep the dynamic derivation concise, it will be useful to define various kinematic relations. First, the augmented matrix of velocities is defined as

$$
\boldsymbol{\nu}=\left[\begin{array}{c}
\dot{\mathbf{r}}_{i}^{s i} \\
\boldsymbol{\omega}_{s}^{s i} \\
\boldsymbol{\omega}_{p}^{p i}
\end{array}\right]=\underbrace{\left[\begin{array}{ccc}
\mathbf{1} & \mathbf{0} & \mathbf{0} \\
\mathbf{0} & \mathbf{S}_{s}^{s i} & \mathbf{0} \\
\mathbf{0} & \mathbf{0} & \mathbf{S}_{p}^{p i}
\end{array}\right]}_{\overline{\mathbf{s}}} \underbrace{\left[\begin{array}{c}
\dot{\mathbf{r}}_{i}^{s i} \\
\dot{\boldsymbol{\theta}}^{s i} \\
\dot{\boldsymbol{\theta}}^{p i}
\end{array}\right]}_{\dot{\mathbf{q}}}
$$

where $\overline{\mathbf{S}}$ is the augmented mapping matrix between the Euler angle rates and the angular velocities. It will also be useful to describe the augmented velocities in terms of the independent angular velocities using

$$
\nu=\underbrace{\left[\begin{array}{cc}
-r \mathbf{1}_{3}^{\times} \mathbf{C}_{s i}^{\top} & \mathbf{0} \\
\mathbf{1} & \mathbf{0} \\
\mathbf{C}_{p s} & \mathbf{1}_{2}
\end{array}\right]}_{\boldsymbol{\Pi}} \underbrace{\left[\begin{array}{c}
\boldsymbol{\omega}_{s}^{s i} \\
\omega_{p, 2}^{p s}
\end{array}\right]}_{\hat{\boldsymbol{\omega}}}
$$

where $\boldsymbol{\Pi}$ is a mapping matrix between the independent angular velocities $\hat{\boldsymbol{\omega}}$ and the augmented velocities $\boldsymbol{\nu}$. Notice that, because the pendulum is constrained to rotate about $s^{2}$ axis, the term $\omega_{p, 2}^{p s}$ is equal to $\dot{\phi}$. To describe the equations of motion of the rover, the time derivative of $\boldsymbol{\Pi}$, given by

$$
\dot{\mathbf{\Pi}}=\left[\begin{array}{cc}
-r \mathbf{1}_{3}^{\times} \mathbf{C}_{s i}^{\top} \boldsymbol{\omega}_{s}^{s i^{\times}} & \mathbf{0} \\
\mathbf{0} & \mathbf{0} \\
-\omega_{p, 2}^{p s} \mathbf{1}_{2}^{\times} \mathbf{C}_{p s} & \mathbf{0}
\end{array}\right]
$$

will be required. Finally, it will prove helpful to expand and rewrite the term $\mathbf{Y}^{\top} \overline{\mathbf{S}}^{\top}$ as

$$
\mathbf{\Upsilon}^{\top} \overline{\mathbf{S}}^{\top}=\left[\begin{array}{rr}
\mathbf{S}_{s}^{s i^{\top}} & \mathbf{0} \\
\mathbf{0} & \mathbf{1}
\end{array}\right] \underbrace{\left[\begin{array}{ccc}
r \mathbf{C}_{s i} \mathbf{1}_{3}^{\times} & \mathbf{1} & \mathbf{C}_{p s}^{\top} \\
\mathbf{0} & \mathbf{0} & \mathbf{1}_{2}^{\top}
\end{array}\right]}_{\boldsymbol{\Delta}}
$$

The kinetic energy of the system is given by

$$
\begin{aligned}
T & =\frac{1}{2}\left[\begin{array}{lll}
\dot{\mathbf{r}}_{i}^{s i^{\top}} & \boldsymbol{\omega}_{s}^{s T^{\top}} & \boldsymbol{\omega}_{p}^{p i^{\top}}
\end{array}\right] \\
& \times \underbrace{\left[\begin{array}{ccc}
\left(m_{\mathcal{S}}+m_{\mathcal{P}}\right) \mathbf{1} & \mathbf{0} & -\mathbf{C}_{p i}^{\top} \mathbf{c}_{p}^{\mathcal{P} p^{\times}} \\
\mathbf{0} & \mathbf{J}_{s}^{\mathcal{S}} & \mathbf{0} \\
\mathbf{c}_{p}^{\mathcal{P} p^{\times}} \mathbf{C}_{p i} & \mathbf{0} & \mathbf{J}_{p}^{\mathcal{P} p}
\end{array}\right]}_{\mathbf{M}} \\
& \times\left[\begin{array}{c}
\boldsymbol{\omega}_{s}^{s i} \\
\boldsymbol{\omega}_{p}^{p i}
\end{array}\right]=\frac{1}{2} \nu^{\top} \mathbf{M} \boldsymbol{\nu}
\end{aligned}
$$

where $\mathbf{M}=\mathbf{M}^{\top}>\mathbf{0}$ is the total mass matrix of the system. The potential energy associated with the rolling tumbleweed rover can be expressed as

$$
U=g m_{\mathcal{P}} \mathbf{1}_{3}^{\top} \mathbf{r}_{i}^{s i}+g \mathbf{1}_{3}^{\top} \mathbf{C}_{p i}^{\top} \mathbf{c}_{p}^{\mathcal{P} p}
$$

Recall Lagrange's Equation ([27] p. 250):

$$
\frac{\mathrm{d}}{\mathrm{d} t}\left(\frac{\partial L}{\partial \dot{\mathbf{q}}}\right)^{\top}-\left(\frac{\partial L}{\partial \mathbf{q}}\right)^{\top}=\boldsymbol{\Xi}^{\top} \lambda+\boldsymbol{f}
$$

where the Lagrangian is defined as $L=T-U, \boldsymbol{\Xi}$ is a constraint matrix satisfying $\boldsymbol{\Xi} \dot{\mathbf{q}}=\mathbf{0}, \boldsymbol{\lambda}$ is a column matrix of the Lagrange multiplier terms, and $\boldsymbol{f}$ is a column matrix of the generalized forces and torques acting on the system. The equations of motion in terms of the angular velocities are

$$
\hat{\mathbf{M}} \dot{\hat{\boldsymbol{\omega}}}+\hat{\boldsymbol{\tau}}^{\text {non }}=\hat{\boldsymbol{\tau}}^{\mathrm{ext}}
$$

where 


$$
\begin{aligned}
& \hat{\mathbf{M}}=\Delta \mathbf{M \Pi}, \\
& \hat{\boldsymbol{\tau}}^{\text {non }}=\boldsymbol{\Delta}\left(\mathbf{M} \dot{\boldsymbol{\Pi}} \hat{\boldsymbol{\omega}}+\boldsymbol{\nu}^{\times} \mathbf{M} \boldsymbol{\nu}+\mathbf{a}\right), \quad \text { and } \quad \hat{\boldsymbol{\tau}}^{\mathrm{ext}}=\boldsymbol{\Delta} \boldsymbol{\tau}^{\mathrm{ext}}
\end{aligned}
$$

The term $\tau^{\text {ext }}$ contains the external forces and torques applied on the system, as described in Sec. IV.A. The matrix $\mathbf{a}$ is given by

$$
\mathbf{a}=\left[\begin{array}{c}
-\mathbf{C}_{p i}^{\top} \boldsymbol{\omega}_{p}^{p i^{\times}} \mathbf{c}_{p}^{\mathcal{P} p^{\times}} \boldsymbol{\omega}_{p}^{p i}+\underset{\mathcal{g} m_{\mathcal{P}} \mathbf{1}_{3}+\dot{\mathbf{r}}_{i}^{s i^{\times}} \mathbf{C}_{p i}^{\top} \mathbf{c}_{p}^{\mathcal{P} p^{\times}} \boldsymbol{\omega}_{p}^{p i}}{\mathbf{0}} \\
-\boldsymbol{\omega}_{p}^{p i^{\times}} \mathbf{c}_{p}^{\mathcal{P} p^{\times}} \mathbf{C}_{p i} \dot{\mathbf{r}}_{i}^{s i}-\left(\mathbf{C}_{p i} \mathbf{1}_{3}\right)^{\times} \mathbf{c}_{p}^{\mathcal{P} p} g
\end{array}\right]
$$

and the term $\nu^{\times}$is defined as

$$
\boldsymbol{\nu}^{\times}=\left[\begin{array}{ccc}
\dot{\mathbf{r}}_{i}^{s i^{\times}} & \mathbf{0} & \mathbf{0} \\
\mathbf{0} & \boldsymbol{\omega}_{s}^{s i^{\times}} & \mathbf{0} \\
\mathbf{0} & \mathbf{0} & \boldsymbol{\omega}_{p}^{p i^{\times}}
\end{array}\right]
$$

This concludes the review of the equations of motion of the tumbleweed rover (for more details, see [12]).

\section{References}

[1] Grotzinger, J. P., Crisp, J., Vasavada, A. R., Anderson, R., Baker, C. J., Barry, R., Blake, D. F., Conrad, P., Edgett, K. S., Ferdowski, B., Gellert, R., Gilbert, J. B., Golombek, M., Gmez-Elvira, J., Hassler, D. M., Jandura, L., Litvak, M., Mahaffy, P., Maki, J., Meyer, M., Malin, M. C., Mitrofanov, I., Simmonds, J. J., Vaniman, D., Welch, R. V., and Wiens, R. C., "Mars Science Laboratory Mission and Science Investigation," Space Science Reviews, Vol. 170, Nos. 1-4, 2012, pp. 5-56. doi:10.1007/s11214-012-9892-2

[2] Kuhlman, K. R., Behar, A. E., Jones, J., Boston, P., Antol, J., Hajos, G., Kelliher, W., Coleman, M., Crawford, R., Rothschild, L., Buehler, M., Bearman, G., and Wilson, D. W., "Tumbleweed: A New Paradigm for Surveying Mars for in Situ Resources," Proceedings of the 12th International Conference on Engineering, Science, Construction, and Operations in Challenging Environments - Earth and Space, Curran Associates, Inc., Red Hook, NY, March 2010, pp. 1502-1512.

[3] Antol, J., Calhoun, P., Flick, J., Hajos, G., Kolacinski, R., Minton, D., Owens, R., and Parker, J., "Low Cost Mars Surface Exploration: The Mars Tumbleweed," NASA TM-2003-212411, Aug. 2003.

[4] Behar, A., Carsey, F., Matthews, J., and Jones, J., "Antarctic Deployment of the NASA/JPL Tumbleweed Polar Rover," Robotics: Trends, Principles, and Applications - Proceedings of the Sixth Biannual World Automation Congress, IEEE Publ., Piscataway, NJ, JuneJuly 2004, pp. 453-460.

[5] Antol, J., Harris, S. B., and Hajos, G. A., "Wind Tunnel Tests of Evolved Mars Tumbleweed Concepts," 44th AIAA Aerospace Sciences Meeting, Vol. 2, AIAA, Reston, VA, Jan. 2006, pp. 890-899.

[6] Antol, J., Chattin, R. L., Copeland, B. M., and Krizan, S. A., "NASA Langley Mars Tumbleweed Rover Prototype," 44th AIAA Aerospace Sciences Meeting, Vol. 2, AIAA, Reston, VA, Jan. 2006, pp. 842-847.

[7] Rose, S. E., Moody, C. B., James, D. L., and Barhorst, A. A., "Drag Measurement and Dynamic Simulation of Martian Wind-Driven Sensor Platform Concepts," Journal of Fluids and Structures, Vol. 22, No. 1, 2006, pp. 21-43. doi:10.1016/j.jfluidstructs.2005.09.008

[8] Basic, G., "Power-Scavenging Tumbleweed Rover," M.S. Thesis, Univ. of Toronto, Toronto, 2010.

[9] Hartl, A. E., and Mazzoleni, A. P., "Parametric Study of Spherical Rovers Crossing a Valley," Journal of Guidance, Control, and Dynamics, Vol.31, No. 3, 2008, pp. 775-779. doi: $10.2514 / 1.33932$

[10] Hartl, A. E., and Mazzoleni, A. P., "Terrain Modeling and Simulation of a Tumbleweed Rover Traversing Martian Rock Fields," Journal of Spacecraft and Rockets, Vol. 49, No. 2, 2012, pp. 401-412. doi:10.2514/1.A32132
[11] Wilson, J. L., Mazzoleni, A. P., DeJarnette, F. R., Antol, J., Hajos, G. A., and Strickland, C. V., "Design, Analysis, and Testing of Mars Tumbleweed Rover Concepts," Journal of Spacecraft and Rockets, Vol. 45, No. 2, 2008, pp. 370-382. doi: $10.2514 / 1.31288$

[12] Forbes, J. R., Barfoot, T. D., and Damaren, C. J., "Dynamic Modeling and Stability Analysis of a Power-Generating Tumbleweed Rover," Multibody System Dynamics, Vol. 24, No. 4, 2010, pp. 413-439. doi:10.1007/s11044-010-9202-2

[13] Flick, J. J., and Toniolo, M. D., "Preliminary Dynamic Feasibility and Analysis of a Spherical, Wind-Driven (Tumbleweed), Martian Rover," 43rd AIAA Aerospace Sciences Meeting and Exhibit, AIAA, Reston, VA, Jan. 2005, pp. 11901-11912.

[14] Hartl, A. E., and Mazzoleni, A. P., "Dynamic Modeling of a WindDriven Tumbleweed Rover Including Atmospheric Effects," Journal of Spacecraft and Rockets, Vol. 47, No. 3, 2010, pp. 493-502. doi:10.2514/1.45174

[15] Yu, T., Sun, H., Jia, Q., Zhang, Y., and Zhao, W., "Stabilization and Control of a Spherical Robot on an Inclined Plane," Research Journal of Applied Sciences, Engineering and Technology, Vol. 5, No. 6 , Feb. 2013, pp. 2289-2296.

[16] Li, T., Wang, Z., and Ji, Z., "Dynamic Modeling and Simulation of the Internal- and External-Driven Spherical Robot," Journal of Aerospace Engineering, Vol. 25, No. 4, 2012, pp. 636-640. doi:10.1061/(ASCE)AS.1943-5525.0000158

[17] Kayacana, E., Bayraktaroglua, Z., and Saeysa, W., "Modeling and Control of a Spherical Rolling Robot: A Decoupled Dynamics Approach," Robotica, Vol. 30, No. 4, 2012, pp. 671-680. doi:10.1017/S0263574711000956

[18] Schroll, G. C., "Dynamic Model of a Spherical Robot from First Principles," M.S Thesis, Colorado State Univ., Fort Collins, CO, 2010.

[19] Balandin, D. V., Komarov, M. A., and Osipov, G. V., "Motion Control for a Spherical Robot with Pendulum Drive," Journal of Computer and Systems Sciences International, Vol. 52, No. 4, 2013, pp. 650-663. doi:10.1134/S1064230713040047

[20] Chase, R., and Pandya, A., "Review of Active Mechanical Driving Principles of Spherical Robots," Robotics, Vol. 1, No. 1, 2012, pp. 3-23. doi:10.3390/robotics 1010003

[21] Tomik, F., Nudehi, S., Flynn, L. L., and Mukherjee, R., "Design, Fabrication and Control of Spherobot: A Spherical Mobile Robot," Journal of Intelligent and Robotic Systems, Vol. 67, No. 2, 2012, pp. 117-131. doi:10.1007/s10846-012-9652-2

[22] Javadi, A. H., and Mojabi, P., "Introducing August: A Novel Strategy for an Omnidirectional Spherical Rolling Robot," Proceedings of the 2002 IEEE International Conference on Robotics and Automation, Vol. 4, IEEE Publ., Piscataway, NJ, May 2002, pp. 3527-3533.

[23] Bhattacharya, S., and Agrawal, S. K., "Spherical Rolling Robot: A Design and Motion Planning Studies," IEEE Transactions on Robotics and Automation, Vol. 16, No. 6, 2000, pp. 835-839. doi: $10.1109 / 70.897794$

[24] Joshi, V. A., Banavar, R. N., and Hippalgaonkar, R., "Design and Analysis of a Spherical Mobile Robot," Mechanism and Machine Theory, Vol. 45, No. 2, 2013, pp. 130-136. doi:10.1016/j.mechmachtheory.2009.04.003

[25] Bruhn, F. C., Kratz, H., Warell, J., Lagerkvist, C.-I., Kaznov, V., Jones, J. A., and Stenmark, L., "Preliminary Design for a Spherical Inflatable Microrover for Planetary Exploration," Acta Astronautica, Vol. 63, Nos. 5-6, 2008, pp. 618-631. doi:10.1016/j.actaastro.2008.01.044

[26] Hughes, P. C., Spacecraft Attitude Dynamics, 2nd ed., Dover, New York, 2004.

[27] Schaub, H., and Junkins, J. L., Analytical Mechanics of Space Systems, 2nd ed., AIAA, Reston, VA, 2009.

[28] Meirovitch, L., Methods of Advanced Dynamics, McGraw-Hill, New York, 1970, pp. 162-164.

D. Spencer Associate Editor 